The Astrophysical Journal, 633:844-856, 2005 November 10

(C) 2005. The American Astronomical Society. All rights reserved. Printed in U.S.A.

\title{
DARK MATTER AND STELLAR MASS IN THE LUMINOUS REGIONS OF DISK GALAXIES
}

\author{
James Pizagno, ${ }^{1}$ Francisco Prada, ${ }^{2}$ David H. Weinberg, ${ }^{1}$ Hans-Walter Rix, ${ }^{3}$ Daniel Harbeck, ${ }^{3,4}$ \\ Eva K. Grebel, ${ }^{3,5}$ Eric F. Bell, ${ }^{3}$ Jon Brinkmann, $^{6}$ Jon Holtzman, $^{7}$ and Andrew West ${ }^{8}$ \\ Received 2005 April 26; accepted 2005 July 22
}

\begin{abstract}
We investigate the correlations among stellar mass $\left(M_{*}\right)$, disk scale length $\left(R_{d}\right)$, and rotation velocity at 2.2 disk scale lengths $\left(V_{2.2}\right)$ for a sample of 81 disk-dominated galaxies (disk/total $\left.\geq 0.9\right)$ selected from the SDSS. We measure $V_{2.2}$ from long-slit $\mathrm{H} \alpha$ rotation curves and infer $M_{*}$ from galaxy $i$-band luminosities $\left(L_{i}\right)$ and $g-r$ colors. We find logarithmic slopes of $2.60 \pm 0.13$ and $3.05 \pm 0.12$ for the (forward fit) $L_{i}-V_{2.2}$ and $M_{*}-V_{2.2}$ relations, somewhat shallower than most previous studies, with intrinsic scatter of 0.13 and 0.16 dex, respectively. Our direct estimates of the total-to-stellar mass ratio within $2.2 R_{d}$, assuming a Kroupa IMF, yield a median ratio of 2.4 for $M_{*}>10^{10} M_{\odot}$ and 4.4 for $M_{*}=10^{9}-10^{10} M_{\odot}$, with large scatter at a given $M_{*}$ and $R_{d}$. The typical ratio of the rotation speed predicted for the stellar disk alone to the observed rotation speed at $2.2 R_{d}$ is $\sim 0.65$. The distribution of scale lengths at fixed $M_{*}$ is broad, but we find no correlation between disk size and the residual from the $M_{*}-V_{2.2}$ relation, implying that the $M_{*}-V_{2.2}$ relation is an approximately edge-on view of the disk galaxy fundamental plane. Independent of the assumed IMF, this result implies that stellar disks do not, on average, dominate the mass within $2.2 R_{d}$. We discuss our results in the context of infall models where disks form in adiabatically contracted cold dark matter halos. A model with a disk-to-halo mass ratio $m_{d}=0.05$ provides a reasonable match to the $R_{d}-M_{*}$ distribution for spin parameters $\lambda$ ranging from $\sim 0.04$ to 0.08 , and it yields a reasonable match to the mean $M_{*}-V_{2.2}$ relation. A model with $m_{d}=0.1$ predicts overly strong correlations between disk size and $M_{*}-V_{2.2}$ residual. Explaining the wide range of halo-to-disk mass ratios within $2.2 R_{d}$ requires significant scatter in $m_{d}$ values, with systematically lower $m_{d}$ for galaxies with lower $M_{*}$ or lower stellar surface density $\Sigma_{*}$.
\end{abstract}

Subject headings: dark matter — galaxies: kinematics and dynamics — galaxies: photometry

\section{INTRODUCTION}

The tight correlation between luminosity and rotation speed is one of the fundamental characteristics of the disk galaxy population (Tully \& Fisher 1977, hereafter TF77). With stellar population modeling and $\mathrm{H}$ i gas measurements, this correlation can be expressed in terms of stellar mass or total baryonic mass, in place of luminosity (Bell \& de Jong 2001, hereafter BD01; McGaugh et al. 2000). The form and tightness of the TF77 relation are critical tests for theoretical models of galaxy formation (e.g., Cole \& Kaiser 1989; Kauffmann et al. 1993; Cole et al. 1994; Eisenstein \& Loeb 1996; Steinmetz \& Navarro 1999; Avila-Reese et al. 1998; Firmani \& Avila-Reese 2000). In classic models of disk galaxy formation by dissipative gravitational collapse (e.g., Fall \& Efstathiou 1980; Gunn 1983; Dalcanton et al. 1997; Mo et al. 1998, hereafter MMW98), the quantities that determine the disk rotation curve are the concentration parameter of the dark matter halo, the ratio of the disk baryonic mass to the total mass of the

\footnotetext{
1 Department of Astronomy, Ohio State University, 4055 McPherson Laboratory, 140 West 18th Avenue, Columbus, OH 43210.

2 Ramon y Cajal Fellow, Instituto de Astrofisica de Andalucia (CSIC), E-18008 Granada, Spain.

3 Max-Planck-Institut für Astronomie, Koenigstühl 17, D-69117 Heidelberg, Germany.

4 Department of Astronomy, University of Wisconsin, 5534 Sterling Hall, 475 North Charter Street, Madison, WI 53706.

5 Astronomisches Institut, Universität Basel, Venusstrasse 7, CH-4102 Binningen, Switzerland.

6 Apache Point Observatory, 2001 Apache Point Road, P.O. Box 59, Sunspot, NM 88349-0059.

7 Department of Astronomy, New Mexico State University, Box 30001, Department 4500, Las Cruces, NM 88003-8001.

8 Department of Astronomy, University of Washington, Box 351580, Seattle, WA 98195-1580.
}

halo, and the disk scale length, which is determined by its angular momentum. These theoretical models suggest that disk size could be an important additional parameter in disk galaxy correlations (Shen et al. 2002; Dutton et al. 2005). A strong correlation between disk size and TF77 residual is also expected if disks are "maximal" and therefore make a dominant contribution to the observed rotation speed (Courteau \& Rix 1999).

In this paper we investigate the correlations among rotation speed, stellar mass, and scale length in a sample of disk galaxies selected from the Sloan Digital Sky Survey (SDSS; York et al. 2000; Abazajian et al. 2004). We have obtained long-slit optical spectra of these galaxies and used them to extract $\mathrm{H} \alpha$ rotation curves. We use the $g-r$ color of each galaxy to estimate its stellar mass-to-light ratio $M_{*} / L$ and therefore its stellar mass, following the prescription of Bell et al. (2003). We apply bulgedisk decomposition to the SDSS $i$-band images to select a sample of disk-dominated galaxies (disk/total $\geq 0.9$ ) and to measure the disk exponential scale length. We estimate circular velocities at 2.2 disk scale lengths, where the rotation curve of a self-gravitating exponential disk reaches its maximum (Freeman 1970). We use the estimated stellar masses to separate the disk and halo contributions to the total mass within this radius, and we investigate the halo-to-disk ratio as a function of stellar mass and disk scale length. We discuss our results in the context of MMW98-style disk galaxy models.

\section{PHOTOMETRIC AND SPECTROSCOPIC OBSERVATIONS}

The SDSS galaxy redshift survey has an unprecedented combination of large area, depth, and photometric quality, thanks to the combination of a large-format camera (Gunn et al. 1998), high-throughput multiobject spectrographs, careful calibration procedures (Fukugita et al. 1996; Hogg et al. 2001; Smith et al. 
2002), and an efficient series of data reduction and targeting pipelines (Lupton et al. 2001; Stoughton et al. 2002; Strauss et al. 2002; Blanton et al. 2003a; Pier et al. 2003; Ivezić et al. 2004). We have selected a sample of $\sim 200$ galaxies from the main galaxy sample (Strauss et al. 2002) of the SDSS redshift survey for follow-up dynamical study with long-slit $\mathrm{H} \alpha$ spectroscopy. Our full sample covers a representative selection of galaxies in the absolute magnitude range $-18 \geq M_{r} \geq-22$, with no morphological preselection other than an $i$-band isophotal axis ratio cut of $b / a<0.6$, needed to allow accurate inclination corrections to observed rotation velocities. We impose a minimum redshift, $c z \geq 5000 \mathrm{~km} \mathrm{~s}^{-1}$, so that peculiar velocities do not cause large uncertainties in distance (and thus luminosity and size). We adopt a luminosity-dependent maximum redshift of $9000\left(-18 \geq M_{r}>-19.5\right), 11,000\left(-19.5 \geq M_{r}>\right.$ $-20.5)$, and $15,000 \mathrm{~km} \mathrm{~s}^{-1}\left(-20.5 \geq M_{r}\right)$, so that galaxies are spatially resolved and the distribution of absolute magnitudes is roughly flat over the range -18 to -22 . Analysis of the TullyFisher relation and its residuals for this broadly representative galaxy sample, including detailed discussion of the sample definition, spectroscopic observations and data reduction procedures, and rotation curve fits, will be presented by J. Pizagno et al. (2005, in preparation, hereafter P05). Here we summarize the relevant aspects of these procedures and describe the selection of the diskdominated galaxy sample that is analyzed in this paper.

In a series of observing runs between 2001 June and 2004 April, we obtained long-slit spectra covering the $\mathrm{H} \alpha$ wavelength region for a total of 234 galaxies in the velocity and absolute magnitude ranges described above, using the TWIN spectrograph on the Calar Alto $3.5 \mathrm{~m}$ telescope (189 galaxies) and the CCDS on the MDM 2.4 m telescope (45 galaxies). Typical exposure times were 30 minutes at Calar Alto and 60 minutes at MDM, with instrumental setups yielding FWHM resolution $\sim 1.48$ and $\sim 1.93 \AA$, respectively. We obtained usable $\mathrm{H} \alpha$ rotation curves for 170 galaxies, $\sim 70 \%$ of the input sample, with the remainder showing insufficient extended $\mathrm{H} \alpha$ emission or (in a few cases) excessively irregular velocity profiles. We applied the bulge-disk decomposition program GALFIT (Peng et al. 2002) to the $i$-band images of these 170 galaxies, taken from the SDSS corrected frames. Specifically, we fitted each galaxy with a combination of an inclined exponential disk and a bulge with a surface brightness profile exp $\left[-\left(r / r_{S}\right)^{1 / n}\right]$ (Sersic 1968), with the index $n$ constrained to the range $0.5 \geq n \geq 5.0$.

For this paper we select those galaxies with disk-to-total luminosity ratio $f_{d} \geq 0.9$. These systems may not be perfectly described by smooth exponential disks, but the addition of a bulge containing more than $10 \%$ of the light does not allow a better fit. We rejected five galaxies for which the discrepancy between the GALFIT exponential disk position angle and the SDSS isophotal position angle, used for the long-slit observations, would lead to a velocity difference of more than $10 \%$. We confirmed the disk-dominated nature of the remaining 81 galaxies by visual inspection. We note that $f_{d} \geq 0.9$ is a stronger morphological cut than that in most TF77 samples, which also include some galaxies with significant bulges. We adopt the more stringent cut mainly because it allows us to define scale length and velocity measures, $R_{d}$ and $V_{2.2}$, that are insensitive to ambiguities of bulge-disk decomposition. Also, while the bulge formation mechanisms in late-type galaxies are uncertain, our sample gives theoretical modelers a clear target to make predictions for nearly bulgeless galaxies, with an absolute magnitude distribution that is approximately flat in the range $-18 \leq M_{r} \leq-22$. We compare results from this sample to those of our full, morphologically representative sample in P05.

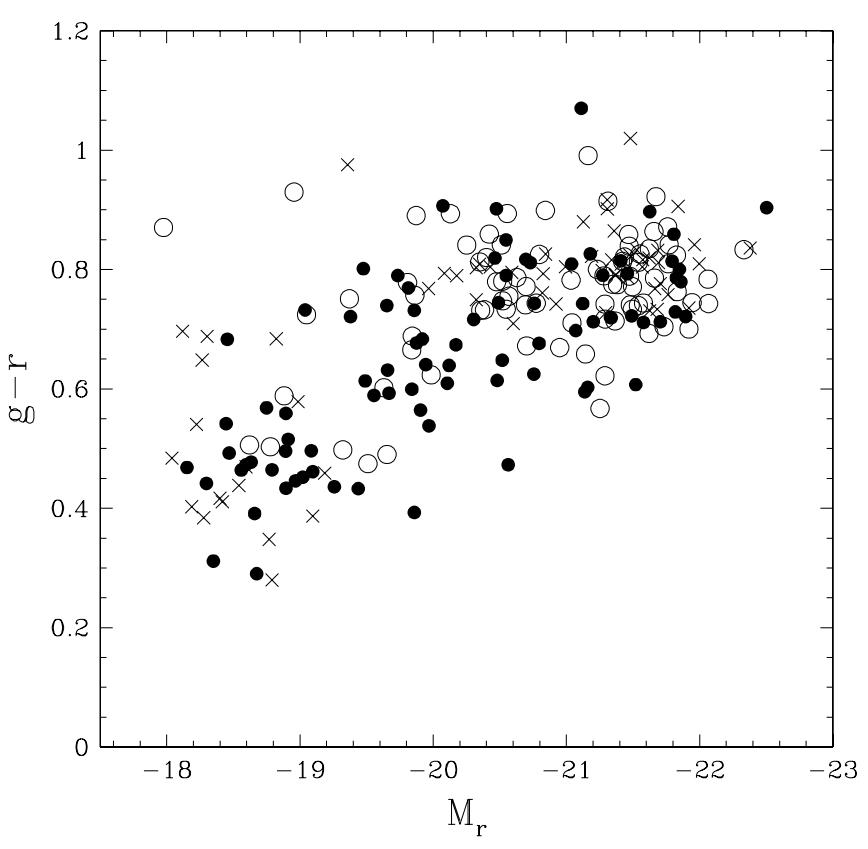

FIG. 1.-Distribution of sample galaxies in the color-absolute magnitude plane. Filled circles are the sample of 81 disk-dominated galaxies analyzed in this paper, and open circles are galaxies with $f_{d}$ less than 0.9. Crosses show galaxies with insufficient extended $\mathrm{H} \alpha$ for useful rotation curve measurements.

We compute galaxy luminosities using SDSS Petrosian fluxes and colors using SDSS model colors, both $K$-corrected to redshift $z=0$ using kcorrect_v3.1b from Blanton et al. (2003b). We compute distances using the SDSS heliocentric redshifts corrected to the rest frame of the Local Group barycenter (Willick et al. 1997), assuming a cosmological model with $\Omega_{m}=0.3$, $\Omega_{\lambda}=0.7$, and Hubble constant $H_{0}=70 \mathrm{~km} \mathrm{~s}^{-1} \mathrm{Mpc}^{-1}$. We incorporate a distance uncertainty corresponding to $300 \mathrm{~km} \mathrm{~s}^{-1}$ when calculating disk scale length and luminosity uncertainties, to account for the typical amplitude of small-scale peculiar velocities (Strauss \& Willick 1995). Figure 1 shows the distribution of our sample galaxies in the color-magnitude plane. Crosses show galaxies that did not have enough $\mathrm{H} \alpha$ emission for extended rotation curves. Although most of these failed galaxies are either red or low luminosity, the galaxies with successful rotation curve measurements cover all populated areas of the colormagnitude plane, so there are no major categories of galaxies in this absolute magnitude range that are missing from our sample. Filled circles show the disk-dominated subset analyzed in this paper. These again span all populated regions of the colormagnitude plane, although the distribution is somewhat bluer than that of the full sample, and the fraction of disk-dominated systems is higher at low luminosity. The surface brightness distribution (not shown) is similar to that of the full sample, although the low- and intermediate-luminosity galaxies that are eliminated by the $f_{d} \geq 0.9$ cut tend to be above the median surface brightness. The GALFIT total magnitudes in $i$ band are 0.12 mag brighter than SDSS Petrosian magnitudes on average, with a standard deviation of $0.10 \mathrm{mag}$, in reasonable agreement with expectations (see Strauss et al. 2002; Graham et al. 2005). We use the Petrosian magnitudes for our analysis so that our results refer to quantities easily accessible from the SDSS database.

The spectroscopic data were dark subtracted, flat-fielded, and linearized using standard IRAF procedures, as outlined by Massey et al. (1992). Following Courteau (1997), we extract two-dimensional spectra along the spatial direction and measure the $\mathrm{H} \alpha$ emission line centroid at each location along the slit. The 
TABLE 1

Galaxy Properties

\begin{tabular}{|c|c|c|c|c|c|c|c|}
\hline SDSS Name & $\begin{array}{c}d \\
(\mathrm{Mpc})\end{array}$ & $\begin{array}{c}L_{i} \\
\left(10^{10} L_{\odot}\right)\end{array}$ & $g-r$ & $\begin{array}{c}M_{*} \\
\left(10^{10} M_{\odot}\right)\end{array}$ & $f_{d}$ & $\begin{array}{c}R_{d} \\
(\mathrm{kpc})\end{array}$ & $\begin{array}{c}V_{2.2} \\
\left(\mathrm{~km} \mathrm{~s}^{-1}\right)\end{array}$ \\
\hline 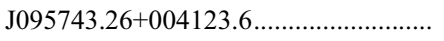 & $200.63(4.43)$ & $4.86(0.86)$ & $0.49(0.06)$ & $5.53(1.07)$ & 0.98 & $4.62(0.10)$ & $196.74(2.87)$ \\
\hline $\mathrm{J} 142729.65+010321.0 \ldots \ldots \ldots \ldots \ldots \ldots \ldots \ldots . .$. & $110.89(4.37)$ & $0.56(0.08)$ & $0.32(0.03)$ & $0.45(0.06)$ & 0.96 & $2.42(0.10)$ & $100.75(3.87)$ \\
\hline 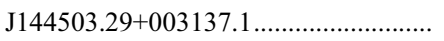 & $124.82(4.38)$ & $2.11(0.32)$ & $0.43(0.04)$ & $2.12(0.35)$ & 0.99 & $4.86(0.17)$ & $157.89(3.10)$ \\
\hline 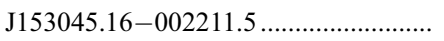 & $166.22(4.41)$ & $4.57(0.71)$ & $0.54(0.06)$ & $5.66(0.95)$ & 0.93 & $3.69(0.10)$ & $198.32(2.58)$ \\
\hline 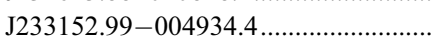 & $106.31(4.36)$ & $0.54(0.10)^{\mathrm{a}}$ & $0.21(0.08)$ & $0.35(0.09)$ & 1.00 & $3.94(0.17)$ & $82.23(3.31)$ \\
\hline J235603.89-000958.6 & $116.31(4.37)$ & $1.02(0.14)$ & $0.25(0.04)$ & $0.71(0.11)$ & 0.97 & $3.58(0.16)$ & $101.82(2.67)$ \\
\hline 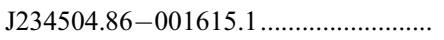 & $103.61(4.36)$ & $1.22(0.23)$ & $0.37(0.06)$ & $1.09(0.23)$ & 0.98 & $4.43(0.19)$ & $116.33(2.56)$ \\
\hline 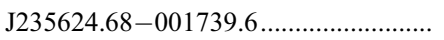 & $110.86(4.37)$ & $0.41(0.05)$ & $0.39(0.03)$ & $0.38(0.05)$ & 1.00 & $2.02(0.08)$ & $93.08(8.01)$ \\
\hline J001006.62-002609.6 …....................... & $143.75(4.39)$ & $2.23(0.31)^{\mathrm{a}}$ & $0.49(0.03)$ & $2.54(0.36)$ & 0.92 & $2.52(0.08)$ & $130.02(2.75)$ \\
\hline 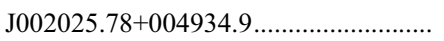 & $76.13(4.34)$ & $2.06(0.34)$ & $0.74(0.02)$ & $3.80(0.63)$ & 0.92 & $5.85(0.34)$ & $127.50(7.68)$ \\
\hline J004239.34+001638.7 .......................... & $195.48(4.43)$ & $5.62(0.59)$ & $0.60(0.03)$ & $7.86(0.87)$ & 0.93 & $6.41(0.15)$ & $246.59(4.01)$ \\
\hline 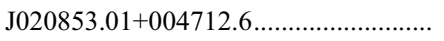 & $188.47(4.42)$ & $7.96(0.99)$ & $0.57(0.04)$ & $10.45(1.38)$ & 0.98 & $6.05(0.14)$ & $245.15(3.87)$ \\
\hline 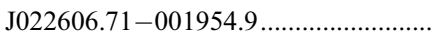 & $94.38(4.35)$ & $7.60(1.23)$ & $0.55(0.05)$ & $9.59(1.64)$ & 0.97 & $7.05(0.33)$ & $251.28(4.03)$ \\
\hline 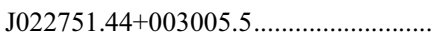 & $182.35(4.42)$ & $10.03(2.41)$ & $0.49(0.10)$ & $11.37(3.06)$ & 0.93 & $9.88(0.25)$ & $251.06(2.78)$ \\
\hline J022820.86+004114.0 & $183.32(4.42)$ & $4.67(0.43)$ & $0.48(0.03)$ & $5.19(0.52)$ & 0.94 & $4.47(0.11)$ & $220.11(3.84)$ \\
\hline 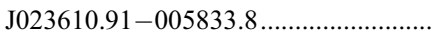 & $220.05(4.44)$ & $8.70(1.54)$ & $0.57(0.06)$ & $11.52(2.17)$ & 0.94 & $5.92(0.12)$ & $252.67(6.44)$ \\
\hline $\mathrm{J} 211450.23-072743.3 \ldots \ldots \ldots \ldots \ldots \ldots \ldots \ldots . .$. & $127.16(4.38)$ & $4.93(0.51)$ & $0.61(0.02)$ & $7.05(0.74)$ & 0.91 & $5.77(0.21)$ & $217.62(4.93)$ \\
\hline J211439.91-075806.9 …………....... & $126.59(4.38)$ & $1.51(0.23)$ & $0.39(0.05)$ & $1.39(0.23)$ & 1.00 & $3.78(0.13)$ & $110.82(3.81)$ \\
\hline $\mathrm{J} 211522.10-074605.0 \ldots \ldots \ldots \ldots \ldots \ldots \ldots \ldots . .$. & $124.79(4.38)$ & $0.41(0.04)$ & $0.42(0.04)$ & $0.41(0.04)$ & 1.00 & $1.22(0.04)$ & $55.98(4.81)$ \\
\hline J001708.77-005728.9 & $84.81(4.35)$ & $0.81(0.10)$ & $0.48(0.03)$ & $0.90(0.12)$ & 1.00 & $3.14(0.16)$ & $133.23(5.56)$ \\
\hline 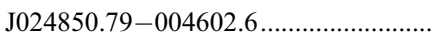 & $95.50(4.35)$ & $5.66(0.96)$ & $0.63(0.05)$ & $8.38(1.47)$ & 0.92 & $4.21(0.19)$ & $200.45(3.09)$ \\
\hline 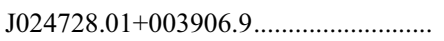 & $199.09(4.43)$ & $4.97(0.66)$ & $0.65(0.01)$ & $7.77(1.03)$ & 0.92 & $3.35(0.08)$ & $204.99(3.95)$ \\
\hline $\mathrm{J} 213703.87-073518.0 \ldots \ldots \ldots \ldots \ldots \ldots \ldots \ldots$ & $131.33(4.38)$ & $1.32(0.15)$ & $0.47(0.03)$ & $1.44(0.18)$ & 0.96 & $2.24(0.08)$ & $135.39(3.62)$ \\
\hline J080658.75+463346.8 & $97.38(4.36)$ & $2.33(0.52)$ & $0.65(0.06)$ & $3.62(0.83)$ & 0.96 & $3.86(0.17)$ & $165.67(3.43)$ \\
\hline 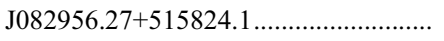 & $77.15(4.34)$ & $0.40(0.06)$ & $0.26(0.04)$ & $0.28(0.05)$ & 1.00 & $3.23(0.18)$ & $83.72(3.39)$ \\
\hline J033329.46-073308.4 ……............... & $76.72(4.34)$ & $0.40(0.06)$ & $0.35(0.03)$ & $0.34(0.06)$ & 1.00 & $2.19(0.12)$ & $97.42(3.83)$ \\
\hline 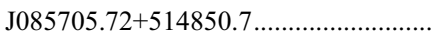 & $76.09(4.34)$ & $0.35(0.06)$ & $0.30(0.04)$ & $0.27(0.05)$ & 0.92 & $5.08(0.32)$ & $107.86(4.80)$ \\
\hline J211816.06-073507.8 …...................... & $128.66(4.38)$ & $7.25(1.20)$ & $0.69(0.05)$ & $12.05(2.07)$ & 0.90 & $5.00(0.17)$ & $228.53(5.76)$ \\
\hline J020045.13-101451.3 ……................... & 81.75 & $0.18(0.02)$ & $0.37(0.02)$ & $0.16(0.02)$ & 1.00 & $2.40(0.13)$ & $77.24(8.71)$ \\
\hline J082949.87+484647.9 & $105.70(4.36)$ & $3.39(0.71)$ & $0.57(0.07)$ & $4.53(1.00)$ & 1.00 & $4.08(0.17)$ & $141.97(2.14)$ \\
\hline $\mathrm{J} 084408.09+504422.9 \ldots \ldots \ldots \ldots \ldots \ldots \ldots \ldots . .$. & $76.21(4.34)$ & $0.69(0.10)$ & $0.51(0.03)$ & $0.80(0.12)$ & 0.99 & $2.66(0.15)$ & $97.89(5.30)$ \\
\hline 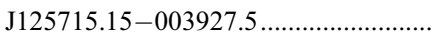 & $98.28(4.36)$ & $1.13(0.17)$ & $0.56(0.06)$ & $1.48(0.25)$ & 0.95 & $1.60(0.07)$ & $138.34(3.25)$ \\
\hline $\mathrm{J} 135433.67-004635.0 \ldots \ldots \ldots \ldots \ldots \ldots \ldots \ldots . .$. & $114.58(4.37)$ & $1.45(0.19)$ & $0.62(0.01)$ & $2.11(0.27)$ & 0.95 & $2.12(0.08)$ & $153.15(3.85)$ \\
\hline 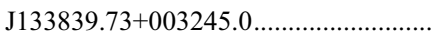 & $97.29(4.36)$ & $0.30(0.04)$ & $0.35(0.03)$ & $0.26(0.03)$ & 0.92 & $4.40(0.21)$ & $106.86(3.83)$ \\
\hline 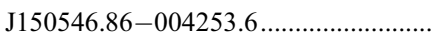 & $155.02(4.40)$ & $2.88(0.40)$ & $0.55(0.02)$ & $3.69(0.52)$ & 0.99 & $4.93(0.14)$ & $174.65(2.50)$ \\
\hline J143842.98-000027.9 & $146.25(4.39)$ & $3.49(0.67)$ & $0.66(0.04)$ & $5.53(1.08)$ & 0.98 & $3.13(0.09)$ & $200.67(5.22)$ \\
\hline 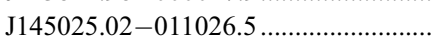 & $187.50(4.42)$ & $7.23(1.40)$ & $0.50(0.08)$ & $8.28(1.81)$ & 0.96 & $5.70(0.14)$ & $244.95(3.27)$ \\
\hline $\mathrm{J} 140452.62-003640.5 \ldots \ldots \ldots \ldots \ldots \ldots \ldots \ldots$ & $106.16(4.36)$ & $3.40(0.36)$ & $0.49(0.02)$ & $3.79(0.41)$ & 0.96 & $5.61(0.23)$ & $185.34(3.80)$ \\
\hline $\mathrm{J} 141413.17-005339.8 \ldots \ldots \ldots \ldots \ldots \ldots \ldots \ldots . .$. & $165.01(4.40)$ & $8.11(1.08)$ & $0.54(0.06)$ & $10.08(1.53)$ & 0.91 & $6.80(0.18)$ & $270.77(3.22)$ \\
\hline J232631.10+005013.5 ……................... & $127.60(4.38)$ & $2.13(0.28)$ & $0.51(0.03)$ & $2.49(0.34)$ & 0.95 & $2.56(0.09)$ & $162.55(2.81)$ \\
\hline J234328.26-000148.6 …...................... & $165.10(4.40)$ & $2.62(0.45)$ & $0.63(0.05)$ & $3.89(0.68)$ & 0.95 & $3.71(0.10)$ & $194.10(3.52)$ \\
\hline J024459.89+010318.5 ........................ & $112.33(4.37)$ & $2.62(0.51)$ & $0.55(0.05)$ & $3.31(0.68)$ & 0.99 & $3.40(0.13)$ & $185.06(3.20)$ \\
\hline 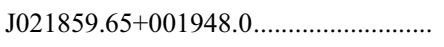 & $132.52(4.38)$ & $3.04(0.49)$ & $0.59(0.04)$ & $4.22(0.69)$ & 0.98 & $3.20(0.11)$ & $197.01(3.68)$ \\
\hline 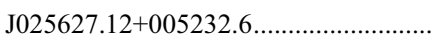 & $101.39(4.36)$ & $3.37(0.39)$ & $0.44(0.05)$ & $3.46(0.48)$ & 0.99 & $5.95(0.26)$ & $160.82(3.86)$ \\
\hline J203523.80-061437.9 …........................... & $85.87(4.35)$ & $4.32(0.64)^{\mathrm{a}}$ & $0.59(0.04)$ & $5.92(0.91)$ & 0.97 & $10.76(0.55)$ & $254.62(2.95)$ \\
\hline J204256.27-065126.1 …....................... & $126.41(4.38)$ & $1.04(0.16)$ & $0.56(0.05)$ & $1.34(0.22)$ & 0.95 & $2.53(0.09)$ & $117.70(5.22)$ \\
\hline $\mathrm{J} 205532.62+000635.6 \ldots \ldots \ldots \ldots \ldots \ldots \ldots \ldots$ & $133.69(4.38)$ & $0.74(0.12)$ & $0.15(0.07)$ & $0.43(0.10)$ & 1.00 & $2.88(0.10)$ & $98.59(2.91)$ \\
\hline $\mathrm{J} 205103.70+000825.5 \ldots \ldots \ldots \ldots \ldots \ldots \ldots \ldots$ & $102.06(4.36)$ & $0.62(0.09)$ & $0.26(0.04)$ & $0.44(0.07)$ & 1.00 & $2.67(0.13)$ & $112.83(10.20)$ \\
\hline
\end{tabular}


TABLE 1 - Continued

\begin{tabular}{|c|c|c|c|c|c|c|c|}
\hline SDSS Name & $\begin{array}{c}d \\
(\mathrm{Mpc})\end{array}$ & $\begin{array}{c}L_{i} \\
\left(10^{10} L_{\odot}\right)\end{array}$ & $g-r$ & $\begin{array}{c}M_{*} \\
\left(10^{10} M_{\odot}\right)\end{array}$ & $f_{d}$ & $\begin{array}{c}R_{d} \\
(\mathrm{kpc})\end{array}$ & $\begin{array}{c}V_{2.2} \\
\left(\mathrm{~km} \mathrm{~s}^{-1}\right)\end{array}$ \\
\hline $\mathrm{J} 215156.74+121411.3 \ldots \ldots \ldots \ldots \ldots \ldots \ldots \ldots \ldots$ & $123.27(4.37)$ & $1.02(0.20)$ & $0.48(0.05)$ & $1.12(0.24)$ & 0.94 & $2.79(0.10)$ & $119.70(5.22)$ \\
\hline $\mathrm{J} 215652.70+121857.5 \ldots \ldots \ldots \ldots \ldots \ldots \ldots \ldots \ldots$ & $135.91(4.38)$ & $3.84(0.44)$ & $0.54(0.04)$ & $4.82(0.58)$ & 0.92 & $5.68(0.19)$ & $184.02(3.71)$ \\
\hline $\mathrm{J} 211343.93+003428.7 \ldots \ldots \ldots \ldots \ldots \ldots \ldots \ldots \ldots$ & $212.02(4.44)$ & $11.12(2.46)$ & $0.57(0.06)$ & $14.75(3.40)$ & 0.97 & $8.07(0.25)$ & $283.32(3.64)$ \\
\hline 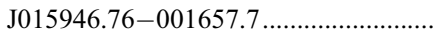 & $194.89(4.43)$ & $5.45(0.65)$ & $0.58(0.04)$ & $7.30(0.91)$ & 0.95 & $4.93(0.11)$ & $241.10(3.92)$ \\
\hline 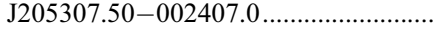 & $132.87(4.38)$ & $0.82(0.09)$ & $0.39(0.03)$ & $0.76(0.09)$ & 0.97 & $3.74(0.17)$ & $114.02(4.56)$ \\
\hline 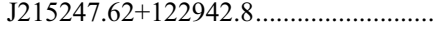 & $129.32(4.38)$ & $0.88(0.09)$ & $0.64(0.02)$ & $1.34(0.13)$ & 0.93 & $3.32(0.12)$ & $124.53(20.89)$ \\
\hline 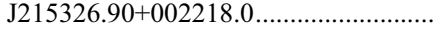 & $123.77(4.37)$ & $1.05(0.12)$ & $0.36(0.04)$ & $0.93(0.12)$ & 0.99 & $5.18(0.19)$ & $135.87(3.83)$ \\
\hline 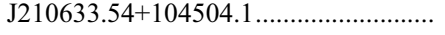 & $126.71(4.38)$ & $1.21(0.14)$ & $0.48(0.03)$ & $1.35(0.17)$ & 1.00 & $3.51(0.12)$ & $108.10(4.02)$ \\
\hline 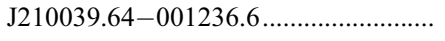 & $107.64(4.36)$ & $0.66(0.12)$ & $0.46(0.06)$ & $0.70(0.14)$ & 0.99 & $3.25(0.16)$ & $102.23(3.00)$ \\
\hline J213811.68+121139.1 ……................... & $99.66(4.36)$ & $1.47(0.22)$ & $0.46(0.05)$ & $1.56(0.26)$ & 0.91 & $2.04(0.09)$ & $99.20(3.43)$ \\
\hline $\mathrm{J} 124545.20+535702.0 \ldots \ldots \ldots \ldots \ldots \ldots \ldots \ldots \ldots$ & $102.17(4.36)$ & $0.36(0.05)$ & $0.26(0.07)$ & $0.25(0.05)$ & 1.00 & $2.78(0.12)$ & $89.83(6.49)$ \\
\hline
\end{tabular}

Notes.-Local Group barycenter distances are in Mpc, for $H_{0}=70 \mathrm{~km} \mathrm{~s}^{-1} \mathrm{Mpc}^{-1}$. Luminosities are derived from SDSS Petrosian magnitudes and corrected for internal dust extinction following Tully et al. (1998). Colors are SDSS model colors corrected for internal extinction. The observed Petrosian magnitudes, model colors, heliocentric redshifts, and corrected frames can be obtained from the public SDSS DR2 server at http://www.sdss.org/dr2.

${ }^{a}$ GALFIT magnitude used instead of SDSS Petrosian because of poor pipeline estimation of the total observed magnitude.

$\mathrm{H} \alpha$ emission line centroid uncertainty is between 2 and $12 \mathrm{~km} \mathrm{~s}^{-1}$ depending on the signal-to-noise ratio of the emission line. We define the rotation curve as the spatial variation of the emissionline centroids along the major axis of the galaxy. We fit galaxy rotation curves with an arctangent function, which has a minimal number of parameters while still describing the global shape of typical rotation curves adequately (Courteau 1997). Specifically, we use a Levenberg-Marquardt $\chi^{2}$ minimization routine (Press et al. 1992) to fit the data with the functional form

$$
V(r)=V_{0}+\frac{2}{\pi} V_{\text {circ }} \arctan \left(\frac{r-r_{0}}{r_{t}}\right)
$$

where $V_{0}$ is the central velocity, $V_{\text {circ }}$ is the asymptotic circular velocity, $r$ is the position along the slit, $r_{0}$ is the center of the rotation curve (where $V=V_{0}$ ), and $r_{t}$ is the turnover radius at which the rotation curve begins to flatten. When performing the fit, we add $10 \mathrm{~km} \mathrm{~s}^{-1}$ in quadrature to the observational error on each $\mathrm{H} \alpha$ data point, to account for noncircular motions and to ensure that parameters are determined by the overall shape of the rotation curve rather than the high signal-to-noise ratio data points in the inner parts of the rotation curve with small uncertainties.

We adopt the circular velocity at 2.2 disk scale lengths as our measure of rotation speed. The rotation curve of an isolated exponential disk peaks at this radius (Freeman 1970), and Courteau (1997) shows that this velocity measure produces the tightest TF77 relation. We infer the observed rotation velocity $\left(V_{2.2}^{\text {obs }}\right)$ by evaluating equation (1) at $r=2.2 R_{d}$, where $R_{d}$ is the $i$-band disk scale length determined by GALFIT. The uncertainty in $V_{2.2}^{\mathrm{obs}}$ is determined using the covariance of the parameter errors returned by the Levenberg-Marquardt routine. We correct $V_{2.2}^{\text {obs }}$ for inclination by using the GALFIT-determined disk axis ratio and the equation

$$
V_{2.2}=V_{2.2}^{\mathrm{obs}}\left[\frac{1-(b / a)^{2}}{1-0.19^{2}}\right]^{-1 / 2}
$$

where 0.19 is the assumed intrinsic axis ratio for an edge-on disk and $b / a$ is the measured $i$-band axis ratio. Observational estimates of the intrinsic axis ratio vary from 0.10 to 0.20 depending on galaxy type (Haynes \& Giovanelli 1984). We choose 0.19, typical for spiral galaxies, and note that the range $0.10-0.20$ corresponds to a small variation (typically $\sim 1 \mathrm{~km} \mathrm{~s}^{-1}$ ) in $V_{2.2}$. As discussed in detail by P05, roughly $\frac{1}{3}$ of our galaxies have rotation curves that are still rising at the outermost $\mathrm{H} \alpha$ point. We have included these galaxies in our sample, but we have checked that excluding them makes minimal difference to our results.

Table 1 lists the SDSS identifier, distance, $i$-band luminosity, $g-r$ color, stellar mass, GALFIT disk fraction, disk exponential scale length, and rotation velocity $V_{2.2}$ for the 81 galaxies that comprise our disk-dominated sample. The luminosities and colors are corrected for internal extinction, and stellar masses are computed from these extinction-corrected quantities, as described in the next section.

\section{RESULTS}

Figure $2 a$ shows the $i$-band TF77 relation for our sample of 81 disk-dominated galaxies. Following standard practice, we correct luminosities and colors for internal extinction based on the disk axis ratio and luminosity, using the prescription of Tully et al. (1998) interpolated to the central wavelength of the SDSS $i$ band. We convert luminosities to solar units using $M_{i, \odot}=4.56$ (Bell et al. 2003). The three representative error crosses in the lower right corner show the 90th, 50th, and 10th percentile values of the observational uncertainties. We add $\frac{1}{3}$ of the inclination correction in quadrature to the luminosity uncertainty to represent the uncertainty in the inclination correction itself. Luminosity uncertainties are dominated by this inclination correction uncertainty and by the $300 \mathrm{~km} \mathrm{~s}^{-1}$ peculiar velocity uncertainty.

The solid line shows our "forward" fit to the observed TF77 relation. Specifically, we fit a relation

$$
y=a\left(x-x_{0}\right)+b,
$$



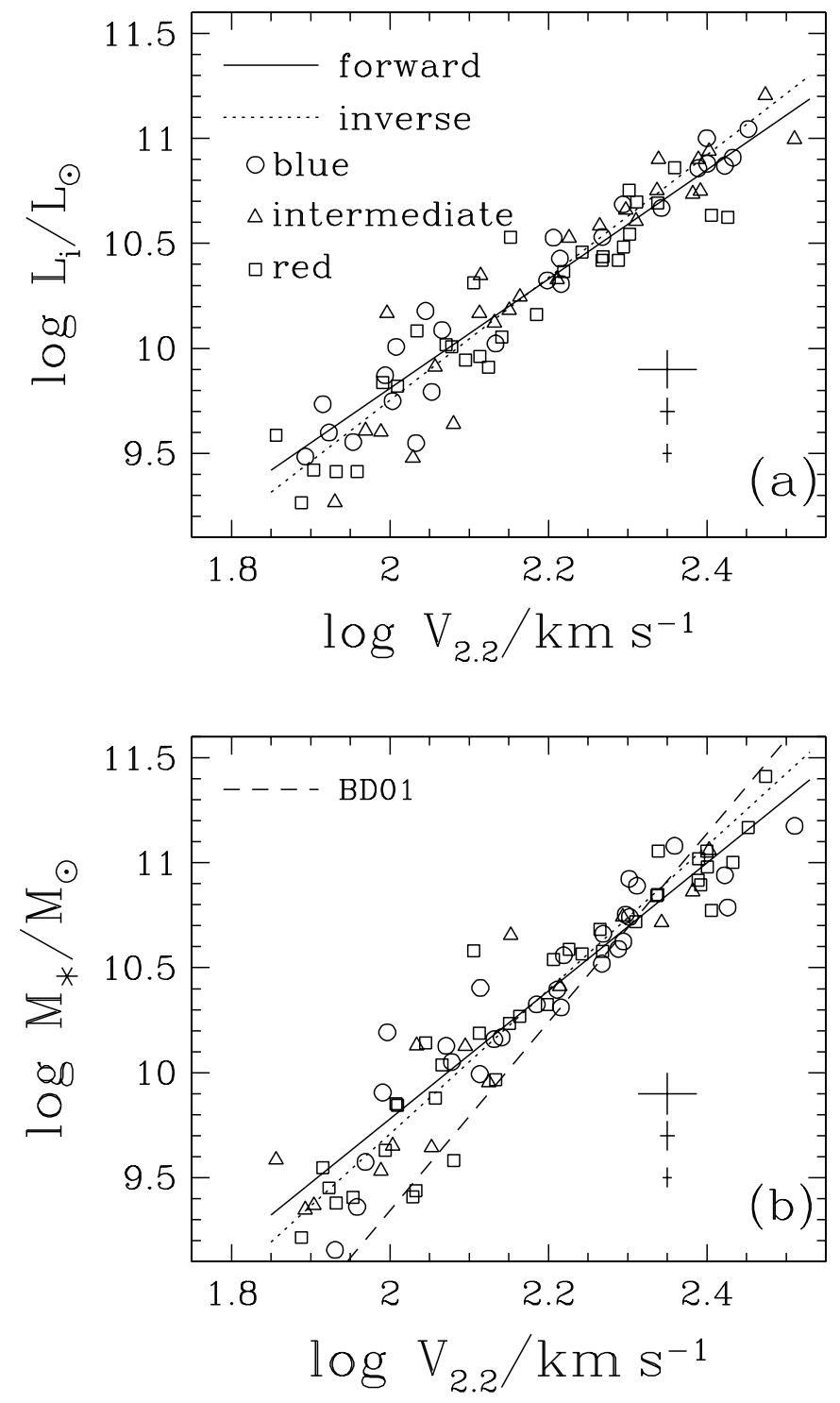

FIG. 2.-(a) TF77 relation of our disk-dominated sample: inclinationcorrected $i$-band luminosity vs. circular velocity at 2.2 disk scale lengths. Circles, triangles, and squares show galaxies that are blue, intermediate, and red, respectively, with respect to the mean color-absolute magnitude relation. Solid and dotted lines show the best forward and inverse fits to the data points, respectively (see Table 2 for parameters). (b) Same as $(a)$, but with $L_{i}$ replaced by the estimated stellar mass $M_{*}$. Here and in subsequent figures, $M_{*}$ is estimated from the $i$-band luminosity and a stellar mass-to-light ratio inferred from the $g-r$ color following Bell et al. (2003; see eq. [6]). The short-dashed line shows the best-fit stellar mass TF77 relation from BD01, adjusted to the Kroupa IMF. In this and all subsequent figures, error crosses show the 90th, 50th, and 10th percentile values of the observational errors.

with $y=\log \left(L_{i} / L_{\odot}\right), x=\log \left(V_{2.2} / \mathrm{km} \mathrm{s}^{-1}\right)$, assuming a Gaussian intrinsic scatter of dispersion $\sigma$ in $y$ at fixed $x$, in addition to observational uncertainties. We determine maximum likelihood values of $a, b$, and $\sigma$ by maximizing

$$
\begin{aligned}
\ln (L)= & -\frac{1}{2} \sum_{i} \ln \left(\sigma^{2}+\sigma_{i, y}^{2}+a^{2} \sigma_{i, x}^{2}\right) \\
& -\sum_{i} \frac{\left(a x_{i}+b-y_{i}\right)^{2}}{2\left(\sigma^{2}+\sigma_{i, y}^{2}+a^{2} \sigma_{i, x}^{2}\right)}+\text { const }
\end{aligned}
$$

where $\sigma_{i, x}$ and $\sigma_{i, y}$ are the observational uncertainties for data point $i$ (see P05 for discussion). We determine the $1 \sigma$ errors on $a, b$, and $\sigma$ by repeating this procedure for 100 bootstrap subsamples of the full data set and taking the dispersion as the uncertainty. We choose the reference value $x_{0}$ so that there is essentially no covariance between the errors in the slope $a$ and intercept $b$.

For the forward TF77 relation, we obtain

$$
\left(\frac{L_{i}}{10^{10} L_{\odot}}\right)=1.84( \pm 0.09)\left(\frac{V_{2.2}}{149.6 \mathrm{~km} \mathrm{~s}^{-1}}\right)^{2.60 \pm 0.13}
$$

The intrinsic scatter is $\sigma=0.13 \mathrm{dex}=0.33 \mathrm{mag}$, comparable to that of other TF77 studies (e.g., Courteau 1997; Kannappan et al. 2002). The dotted line shows the inverse TF77 fit, in which we assume that there is Gaussian intrinsic scatter of $\log V_{2.2}$ at fixed $\log L_{i}$ instead of the reverse. Forward and inverse fits correspond to different hypotheses about the intrinsic distribution of the correlated quantities, and they yield different slopes except in the limit of zero intrinsic scatter. Our inclusion of the intrinsic scatter as a fit parameter means that points with small observational errors do not get inappropriately large weights in determining the slope and normalization, a difference from many previous analyses. However, for this sample the intrinsic scatter is small enough that the derived slope is not highly sensitive to the fitting procedure (for example, the inverse fit is $\left.V_{2.2} \propto L_{i}^{1 / 2.9}\right)$. Our slope is shallower than that found by some previous studies, e.g., Verheijen (2001), who finds a slope of $\approx 4.5$ in $K^{\prime}$ band, or Kannappan et al. (2002), who find a slope of $\approx 4.0$ in $R$ band. However, it agrees well with the $r$-band slope of 2.54 found by Courteau (1997), whose sample criteria and analysis procedures are most similar to ours. We discuss possible contributions to slope differences in more detail below. Our estimates of the slopes, intercepts, and intrinsic scatter of these and all other bivariate relations fitted in this paper are listed in Table 2. Residuals from these relations show no discernible correlation with axis ratio, which indicates that our inclination corrections are accurate in the mean, even if they are not perfect on a galaxy-by-galaxy basis. The Appendix presents a Monte Carlo test for Malmquist-type biases in our sample selection and analysis and shows that they are small compared to our quoted statistical errors, with an effect of $\sim 0.01$ on the forward TF77 slope.

The point types in Figure $2 a$ encode galaxy color, relative to the expectation for the galaxy's $i$-band luminosity. We fit a linear mean relation to the sample's $(g-r)$ versus $\log L_{i}$ relation and divide the sample into three nearly equal parts based on the residual from this relation. Squares represent galaxies redder than the mean by 0.02 mag or more, circles represent galaxies bluer by $0.05 \mathrm{mag}$ or more, and triangles show the remaining galaxies. There is a slight tendency for red galaxies to lie below the mean TF77 relation, as one might expect given the higher mass-to-light ratios or red stellar populations, but the trend is weak relative to the scatter.

In Figure $2 b$ we have converted galaxy $i$-band luminosities to stellar masses, using the prescription of Bell et al. (2003) to infer each galaxy's stellar mass-to-light ratio from its $(g-r)$ color. We use color in preference to the spectroscopic methods of Kauffmann et al. (2003) because the SDSS fibers cover only the central regions of these relatively nearby galaxies and may not sample a representative stellar population. Bell et al. (2003) adopt a "diet Salpeter" initial mass function (IMF) chosen so that stellar disks have the maximum mass allowed by rotation curve constraints. At each $g-r$ color, we multiply their stellar mass-to-light ratios by 0.71 to correspond to a Kroupa IMF, 
TABLE 2

Bivariate Relation Fits

\begin{tabular}{llccrc}
\hline \hline \multicolumn{1}{c}{$y$} & \multicolumn{1}{c}{$x$} & $x_{0}$ & $a( \pm)$ & $b( \pm)$ & $\sigma( \pm)$ \\
\hline $\log L_{i}$ & $\log V_{2.2}$ & 2.175 & $2.603(0.133)$ & $10.266(0.020)$ & $0.131(0.015)$ \\
$\log V_{2.2}$ & $\log L_{i}$ & 10.293 & $0.342(0.016)$ & $2.185(0.006)$ & $0.048(0.005)$ \\
$g-r$ & $\log L_{i}$ & 10.293 & $0.179(0.013)$ & $0.479(0.009)$ & $0.073(0.010)$ \\
$g-r$ & $\log M_{*}$ & 10.345 & $0.171(0.012)$ & $0.499(0.008)$ & $0.061(0.008)$ \\
$\log M_{*}$ & $\log V_{2.2}$ & 2.192 & $3.048(0.121)$ & $10.365(0.018)$ & $0.158(0.021)$ \\
$\log V_{2.2}$ & $\log M_{*}$ & 10.345 & $0.291(0.011)$ & $2.186(0.006)$ & $0.049(0.007)$ \\
$\log R_{d}$ & $\log M_{*}$ & 10.345 & $0.242(0.030)$ & $0.588(0.012)$ & $0.142(0.011)$ \\
$\log V_{*, 2.2} \mathrm{a}$ & $\log V_{2.2}$ & 2.192 & $1.157(0.053)$ & $2.000(0.008)$ & $0.057(0.008)$ \\
$\log V_{2.2}$ & $\log V_{*, 2.2} \mathrm{a}$ & 1.93 & $0.765(0.042)$ & $2.138(0.007)$ & $0.047(0.007)$ \\
$\log V_{*, 2.2} \mathrm{~b}$ & $\log V_{2.2}$ & 2.192 & $1.128(0.085)$ & $2.000(0.011)$ & $0.105(0.014)$ \\
$\log V_{2.2}$ & $\log V_{*, 2.2}^{\mathrm{b}}$ & 1.93 & $0.661(0.050)$ & $2.143(0.010)$ & $0.081(0.011)$ \\
\hline
\end{tabular}

Notes.-Bivariate relations are fitted with the model $y=a\left(x-x_{0}\right)+b$ with a Gaussian intrinsic scatter of $y$ at fixed $x$, with dispersion $\sigma$. Errors listed for $a, b$, and $\sigma$ are computed from 100 bootstrap trials, and the value of $x_{0}$ is chosen so that errors in $a$ and $b$ are uncorrelated. Luminosities are in $L_{i, \odot}$, stellar masses in $M_{\odot}$, velocities in $\mathrm{km} \mathrm{s}^{-1}$, and radii in $\mathrm{kpc}$.

${ }^{\text {a }} V_{*, 2.2}$ is computed using the mean disk scale length for the galaxy's stellar mass.

b $V_{*, 2.2}$ is computed using each galaxy's measured scale length.

which better represents direct observational estimates of the IMF (for further discussion see Bell et al. 2003). Specifically, we calculate stellar masses using the relations

$$
\begin{gathered}
M_{*} / M_{\odot}=\left(L_{i} / L_{\odot}\right)\left(M_{*} / L_{i}\right), \\
\log \left(M_{*} / L_{i}\right)=-0.222+0.864(g-r)+\log 0.71,
\end{gathered}
$$

where the two coefficients are from Table 7 of Bell et al. (2003). We use inclination-corrected luminosities and colors, again based on the Tully et al. (1998) prescriptions, but the inclination effects are small (typically less than $10 \%$ ) because galaxies move along a locus of roughly constant $M_{*}$ as their luminosities and colors are corrected for extinction. We add an error contribution to $M_{*}$ that is one-third of the applied inclination correction.

Points in Figure $2 b$ are again coded by galaxy $g-r$ color residual, now computed as a function of stellar mass. The conversion to stellar mass has removed the slight trend of TF77 residual with color residual, as one might expect if stellar mass is the more fundamental quantity. Solid and dotted lines show our best-fit forward and inverse relations, respectively, which are listed in Table 1. The forward relation is

$$
\left(\frac{M_{*}}{10^{10} M_{\odot}}\right)=2.32( \pm 0.10)\left(\frac{V_{2.2}}{155.6 \mathrm{~km} \mathrm{~s}^{-1}}\right)^{3.05 \pm 0.12}
$$

The best-fit intrinsic scatter is 0.16 dex, slightly higher than the 0.13 dex found for the $L_{i}-V_{2.2}$ relation. Some of this increase could reflect galaxy-to-galaxy variations in stellar populations or extinction properties, which would change the true $M_{*} / L$ ratios at fixed $g-r$ color; we have assumed a deterministic relation between $g-r$ and $M_{*} / L$ and have not included any scatter about this relation in our observational error budget. The BD01 models have 0.1 dex scatter in stellar mass-to-light ratio $\left(M_{*} / L_{i}\right)$ at fixed color.

The short-dashed line shows the best-fit stellar mass TF77 relation found by BD01, using Verheijen's (2001) data for the Ursa Major Cluster. We have multiplied the normalization of their relation (the $I$-band fit with mass-dependent inclination correction from their Table 2) by 0.71 to adjust to the Kroupa IMF assumed here. Distances are calibrated to $H_{0} \approx 70 \mathrm{~km} \mathrm{~s}^{-1}$ $\mathrm{Mpc}^{-1}$ in both cases, although there could be some uncertainty in the relative distance normalization from the peculiar velocity of Ursa Major. The two relations agree at $V_{2.2} \sim 200 \mathrm{~km} \mathrm{~s}^{-1}$, but the BD01 relation is substantially steeper than ours, with a slope of $4.49 \pm 0.23$ versus $3.05 \pm 0.12$. At $V_{2.2} \sim 100 \mathrm{~km} \mathrm{~s}^{-1}$ the BD01 relation traces the lower envelope of our data points and lies $\sim 0.4$ dex below our best-fit relations. Differences in the samples and analysis methods include the following: our use of the updated Bell et al. (2003) stellar population models in place of the BD01 models, our use of a disk/total $\geq 0.9$ cut versus BD01's more generic "late-type galaxy" selection, our use of $V_{2.2}$ as a velocity measure in place of Verheijen's (2001) $V_{\text {flat }}$ measure used by BD01, and our "field" (or, more accurately, random) environment selection versus their cluster sample,

Since Verheijen (2001) finds a steep $K^{\prime}$-band TF77 relation for the Ursa Major galaxies, BD01's steep slope (relative to ours) appears to be intrinsic to the sample, not a consequence of any differences in stellar population modeling. Our disk/total cut also seems unlikely to be the main source of difference, since the low- $V_{2.2}$ galaxies that are in our sample lie significantly above any of the BD01 data points, and the relatively small number of low- $V_{2.2}$ galaxies excluded by our cut lies on or above the best-fit relation. To investigate the importance of velocity definition differences, we used the data of Courteau (1997), who lists $V_{2.2}$ and the maximum velocity $V_{\max }$ derived from a five-parameter fit to optical rotation curves. The typical ratio of $V_{\max }$ to $V_{2.2}$ is higher for less massive galaxies, and since $V_{\text {flat }}$ is likely to track $V_{\max }$ more closely than $V_{2.2}$, the trend goes in the right direction to explain the discrepancy. However, if we scale up our $V_{2.2}$ values using a mean correction derived from the Courteau (1997) data, then our $M_{*}-V_{2.2}$ slope changes to 3.47, still much shallower than BD01, and the gap between the relations is still $\sim 0.2$ dex in $M_{*}$ at $V_{2.2} \sim 100 \mathrm{~km} \mathrm{~s}^{-1}$. Thus, it appears that velocity definition differences can account for roughly half of the difference between our results and BD01's. We reach a similar conclusion by comparing Verheijen's (2001) $r$-band TF77 data points to our own (see P05). The most plausible source we can identify for the remaining gap is a systematic difference in properties of field and cluster spiral galaxies at low luminosity. Reconciling the two measurements requires the cluster galaxies to rotate faster by $\sim 0.1-0.2$ dex at fixed $M_{*}$. Fully addressing this difference requires a large sample with a range of environments and both $\mathrm{H}$ I and optical data, so that one 
can mimic selection and analysis procedures used by different authors.

Our stellar mass TF77 relation is also shallower than the baryonic TF77 relation derived by McGaugh et al. (2000), $M_{\mathrm{bar}} / 10^{10} M_{\odot}=$ $2.12\left(V / 155.6 \mathrm{~km} \mathrm{~s}^{-1}\right)^{3.98}$ (for $H_{0}=70 \mathrm{~km} \mathrm{~s}^{-1} \mathrm{Mpc}^{-1}$ ). Here $M_{\mathrm{bar}}$ is the sum of the stellar mass and the gas mass inferred from H I measurements. We cannot directly estimate $M_{\text {bar }}$ for our galaxies because we do not have H I data, but Kannappan (2004) reports a statistical correlation (with substantial scatter) between gas-to-stellar mass fraction and SDSS $u-r$ color, $\log (G / S)=$ $1.46-1.06(u-r)$. If we apply this correction to our sample on a galaxy-by-galaxy basis, we obtain $M_{\text {bar }} / 10^{10} M_{\odot}=$ $2.86\left(V / 155.6 \mathrm{~km} \mathrm{~s}^{-1}\right)^{2.89}$, shallower than equation (7) because low-mass galaxies have higher gas content. The average mass increase is 0.2 dex at $V_{2.2} \sim 100 \mathrm{~km} \mathrm{~s}^{-1}$ and 0.05 dex at $V_{2.2} \sim$ $200 \mathrm{~km} \mathrm{~s}^{-1}$. Our slope is substantially shallower than that found by McGaugh et al. (2000), who combine several data sets obtained in different bands. ${ }^{9}$ The McGaugh et al. (2000) sample covers a much wider mass range than ours, extending to circular velocities $V \sim 30 \mathrm{~km} \mathrm{~s}^{-1}$. The difference between optical line width $2 V_{2.2}$ and the $\mathrm{H}$ I line width $W_{20}$ used by McGaugh et al. (2000) could become more important at these low velocities, partly explaining the difference in slope, but we do not see an easy way to fully reconcile the results.

Figure 3 plots the GALFIT disk scale length $R_{d}$ against stellar mass. The dotted line shows the best-fit mean relation

$$
R_{d}=3.87( \pm 0.11)\left(\frac{M_{*}}{2.21 \times 10^{10} M_{\odot}}\right)^{0.24 \pm 0.03} \mathrm{kpc}
$$

However, scatter about this mean relation is very broad. Points are coded by the residual from this best-fit relation, with squares, triangles, and circles representing the largest, intermediate, and smallest $\frac{1}{3}$ of the galaxies, respectively, at a given luminosity.

Theoretical expectations for the distribution of disk galaxies in the space of stellar mass, scale length, and circular velocity are clearly described by, e.g., Fall \& Efstathiou (1980), MMW98, Dalcanton et al. (1997), Mo \& Mao (2000), Shen et al. (2002), and Courteau et al. (2003). In this paper we use a modeling approach similar to that of MMW98 to place our observational results in theoretical context. The central solid line in Figure 3 shows the predicted $R_{d}-M_{*}$ relation for galaxies with a ratio $m_{d}=0.05$ of stellar mass to total halo mass formed in an NFW halo (Navarro et al. 1997) with concentration parameter $c=10$ and spin parameter $\lambda=0.06$. We compute this relation using equation (28) of MMW98, which includes the effects of disk self-gravity and adiabatic contraction of the inner regions of the halo. We set the specific angular momentum of the disk equal to that of the halo $\left(j_{d} / m_{d}=1\right.$ in MMW98's notation), so our quantity $\lambda$ is equivalent to their $\lambda^{\prime}$. While the predicted relation is steeper than our best fit, it roughly describes the central trend of our data points. Upper and lower solid lines show the predictions for $\lambda=0.08$ and 0.04 , respectively. The envelope of these lines encloses roughly the central $80 \%$ of the data points.

The distribution of halo spin parameter in $N$-body simulations is approximately lognormal with a mean $\langle\lambda\rangle \approx 0.04$ and dispersion $\sigma_{\ln \lambda} \approx 0.5$ (Bullock et al. 2001). For $m_{d}=0.05$, reproducing the $R_{d}-M_{*}$ relation requires $\lambda$-values in the upper

\footnotetext{
9 McGaugh et al. (2000) also assumed a constant stellar mass-to-light ratio in each band. Had they included a color dependence, they would have derived a still steeper slope, since fainter galaxies are generally bluer and therefore have lower $M_{*} / L$.
}

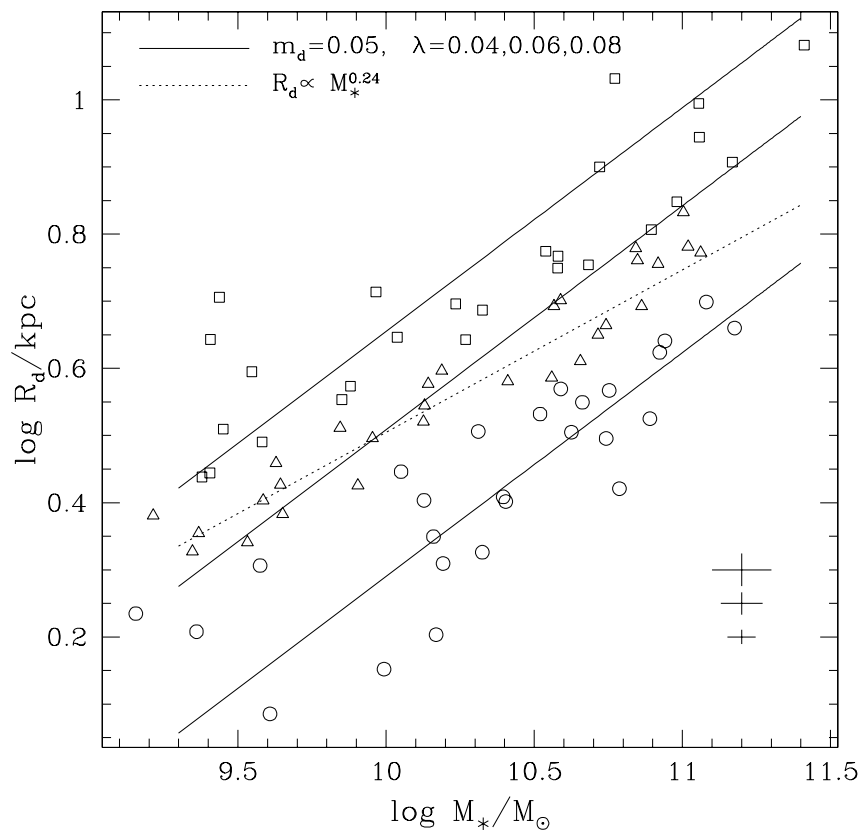

FIG. 3.-Relation between disk scale length and stellar mass. The dotted line is the best-fit mean relation, and points are coded by distance from this relation. The central solid line shows the predicted relation for exponential disks formed in adiabatically contracted NFW halos with concentration $c=10$, spin parameter $\lambda=0.06$, and ratio $m_{d}=0.05$ of disk mass to halo virial mass. Lower and upper solid lines show the model predictions for $\lambda=0.04$ and 0.08 , respectively. Other combinations of $\left(m_{d}, \lambda\right)$ can produce similar results, as discussed in the text.

half of this distribution, so disks would have to either form in the higher spin halos or have a higher specific angular momentum than the dark matter. Since systems with low angular momentum may be more likely to form a substantial bulge and thus be omitted from our disk-dominated sample, this preferential sampling of the high end of the $\lambda$-distribution is not implausible. However, the three solid lines in Figure 3 can also be produced (almost exactly) with a disk-to-halo mass fraction $m_{d}=0.025$ and $\lambda$ of $0.03,0.045,0.06$; the lower $m_{d}$ shifts the predicted relations to lower $M_{*}$, and lower $\lambda$-values are required to compensate. Conversely, for $m_{d}=0.10$, the $\lambda$-values that yield similar $R_{d}-M_{*}$ curves are $0.055,0.08$, and 0.11 .

Figure 4 again shows the stellar mass TF77 relation, $M_{*}$ versus $V_{2.2}$, but points are now coded by their residual from the best-fit $R_{d}-M_{*}$ relation, with squares, triangles, and circles representing the largest, intermediate, and smallest galaxies, respectively, just as in Figure 3. There is no evident separation among these three sets of points, i.e., no tendency of large or small galaxies to lie above or below the mean $M_{*}-V_{2.2}$ relation. A plot of $M_{*}-V_{2.2}$ residual against $R_{d}-M_{*}$ residual (shown as inset) is simply a scatter plot. As noted by Courteau \& Rix (1999) and Courteau et al. (2003), the lack of correlation between TF77 residual and disk scale length argues against the "maximal disk" hypothesis, in which the stellar disk provides a large fraction of the rotational support at $2.2 R_{d}$, since in this case $V_{2.2}^{2} \propto G M_{*} /\left(2.2 R_{d}\right)$. We concur with both their observational result (indeed, our residual correlation appears even weaker) and their conclusion.

Filled squares in Figure 4 show model predictions for disks with mass fraction $m_{d}=0.05$ and the spin parameter $\lambda=0.06$ that yields the central solid line of Figure 3 , with total halo masses of $M_{h}=4 \times 10^{10}, 1.89 \times 10^{11}$, and $1.39 \times 10^{12} M_{\odot}$. (Following MMW98, we define the halo mass within a virial radius whose mean interior density is 200 times the critical 


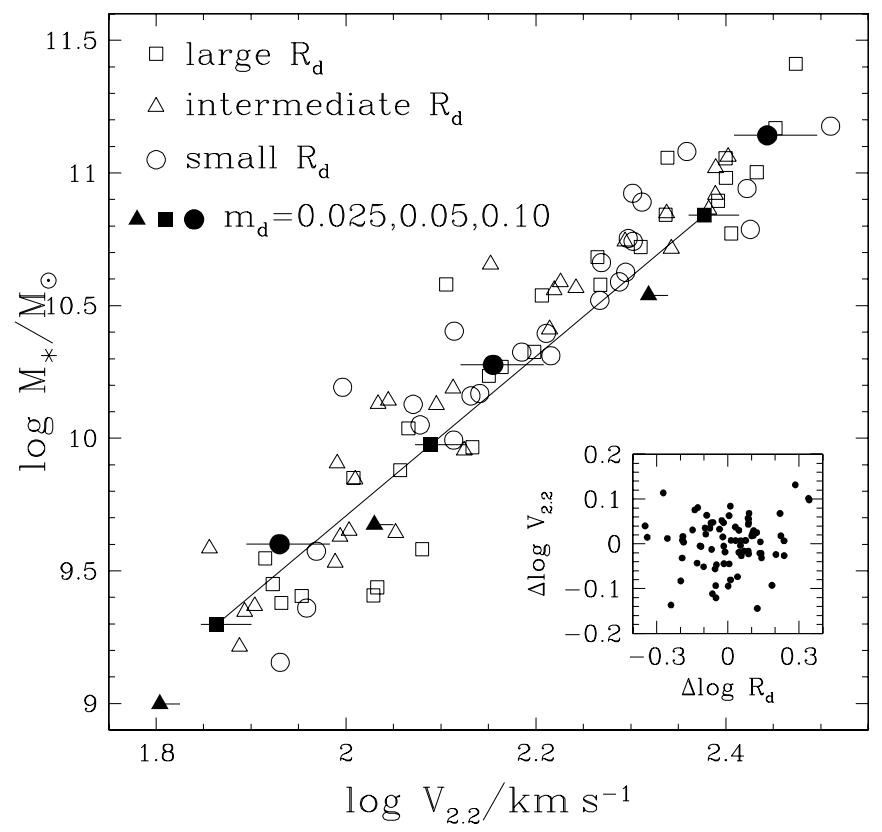

FIG. 4.- Stellar mass TF77 relation, as in Fig. $2 b$, but with points coded by their residual from the mean $R_{d}-M_{*}$ relation, as in Fig. 3 . The inset panel plots TF77 residual against $R_{d}$ residual. In the main panel, filled squares show predictions for a model with $m_{d}=0.05, c=10$, and $\lambda=0.06$, for three different halo masses. Horizontal error bars on these points show the change in $V_{2.2}$ when $\lambda$ is changed to 0.04 (higher $V_{2.2}$ ) or 0.08 (lower $V_{2.2}$ ), the values corresponding roughly to the range of observed disk sizes. Filled circles and filled triangles show corresponding predictions for $m_{d}=0.10$ and 0.025 , respectively, with $\lambda$-values chosen to produce the observed range of disk sizes for the corresponding $m_{d}$ (see text for further discussion).

density.) We again assume an initial NFW halo concentration $c=10$ and compute $R_{d}$ using MMW98's equation (28), but we compute the response of the halo to the disk using the improved adiabatic contraction approximation of Gnedin et al. (2004), with code kindly provided by $\mathrm{O}$. Gnedin. With these parameters, the model reproduces the slope and normalization of our measured $M_{*}-V_{2.2}$ relation, as well as the $R_{d}-M_{*}$ relation. Horizontal lines attached to these points show the effect of changing the spin parameter to $\lambda=0.04$ and 0.08 , corresponding to the lower and upper lines in Figure 3. Larger disks have weaker self-gravity and therefore lower $V_{2.2}$, while compact disks make a substantial contribution to $V_{2.2}$ and therefore spin faster. However, the predicted difference between large and small disks is small enough that it could plausibly be swamped by the scatter seen in Figure 4.

Filled triangles in Figure 4 show models with $m_{d}=0.025$ and $\lambda=0.045$, which also reproduce the central line of Figure 3, for the same three halo masses (and $c=10$ ). Lowering $m_{d}$ reduces both $M_{*}$ and the disk contribution to $V_{2.2}$, but the shift is not exactly parallel to the $M_{*}-V_{2.2}$ relation, so the model predictions for $m_{d}=0.025$ lie below the central trend of the data, by $\sim 0.1-0.2$ dex. However, a choice of IMF with fewer lowmass stars would reduce $M_{*} / L_{i}$ ratios at fixed color and could bring down the data points to agree with the $m_{d}=0.025$ predictions. Because of the lower disk mass fraction, variations of $\lambda$ that reproduce the spread in the $R_{d}-M_{*}$ relation $(\lambda=$ $0.03-0.06)$ produce only small shifts in $V_{2.2}$ at fixed $M_{*}$.

Filled circles show models with $m_{d}=0.10$ and $\lambda=0.08$. The model predictions now lie above the central trend of the data. Adopting a more bottom-heavy IMF could raise the data points and remove this discrepancy, but the $m_{d}=0.10$ model predicts a substantial change of $V_{2.2}$ over the range $\lambda=$
$0.055-0.11$ that reproduces the spread in the $R_{d}-M_{*}$ relation. For $m_{d}=0.10$, the compact galaxies (open circles) should lie noticeably to the right of the large galaxies (open squares) in Figure 4 , and they do not. In other words, $m_{d}=0.10$ disks in adiabatically contracted, $c=10$, NFW halos are too close to maximal to be consistent with the absence of a size-TF77 residual correlation. It is difficult to put this discrepancy in fully quantitative terms because a viable model would have to specify what parameters other than $\lambda$ are varying to produce the intrinsic scatter in the $M_{*}-V_{2.2}$ relation. We will investigate this question in future work.

Figure $5 a$ repackages the information in Figures 3 and 4 by plotting the rotation velocity predicted for the stellar disk,

$$
V_{*, 2.2}=\left(\frac{G M_{*}}{2.2 R_{d}}\right)^{1 / 2}\left[1.32\left(0.65 f_{d}\right)+\left(1-f_{d}\right)\right]^{1 / 2},
$$

against the observed rotation velocity $V_{2.2}$. The factor 0.65 in the brackets is the fraction of the disk mass within $2.2 R_{d}$, and the factor of 1.32 accounts for the flattened geometry of the disk potential (Freeman 1970; Binney \& Tremaine 1987). The $\left(1-f_{d}\right)$ term represents the contribution of the bulge, which we assume to lie entirely within $2.2 R_{d}$; this contribution is small, since $f_{d} \geq 0.9$ for our sample. In Figure $5 a$ we use the mean value of $R_{d}$ at the galaxy's $M_{*}$, from the best-fit relation shown in Figure 3 and listed in Table 1. This plot simply tilts the stellar mass TF77 relation to account for the increase of average disk size with stellar mass. The solid line shows the best-fit (forward) relation,

$\frac{V_{*, 2.2}}{156 \mathrm{~km} \mathrm{~s}^{-1}}=(0.64 \pm 0.01)\left(\frac{V_{2.2}}{156 \mathrm{~km} \mathrm{~s}^{-1}}\right)^{1.16 \pm 0.05}$.

The slope is just slightly steeper than the unit slope predicted for pure self-gravitating disks, but the typical offset is a factor of $\sim 0.65$. Since velocities add in quadrature, $V_{\text {tot }}^{2}=V_{*}^{2}+V_{h}^{2}$, the normalization of equation (9) implies a typical ratio of $V_{h} / V_{*} \sim$ 1.2 of the halo and stellar disk circular velocities at $2.2 R_{d}$. The intrinsic scatter in $V_{*, 2.2}$ at fixed $V_{2.2}$ is $0.057 \mathrm{dex}$. If we apply Kannappan's (2004) color-based estimate of gas-to-stellar mass ratios to compute the total disk contribution $V_{d, 2.2}$ instead of $V_{*, 2.2}$, then the normalization of equation (10) rises slightly, to 0.71 , and the slope changes to 1.07 , a nearly constant ratio of disk mass to dark halo mass within $2.2 R_{d}$.

In Figure $5 b$ we use each galaxy's actual scale length, instead of the mean scale length from the $R_{d}-M_{*}$ relation, when computing $V_{*, 2.2}$. The slope and normalization of the best-fit relation are virtually unchanged, but the intrinsic scatter is nearly 2 times larger, 0.105 dex instead of 0.058 dex. Furthermore, the compact galaxies (circles) lie systematically above the mean relation (high $V_{*, 2.2}$ at a given $V_{2.2}$ ), and the large galaxies (squares) lie systematically below. Thus, even though the slope of the $V_{*, 2.2}-V_{2.2}$ relation is close to unity, the value of $V_{*, 2.2}$ is, on a galaxy-by-galaxy basis, a worse predictor of circular velocity than the stellar mass alone. Dashed lines in Figure 5 show $V_{*, 2.2}=0.85 V_{2.2}$, which Sackett (1997) describes as a good approximation to the standard "maximal disk" hypothesis. ${ }^{10}$

10 Specifically, Sackett (1997) states that in maximal disk decompositions, the disk rotation speed is typically $75 \%-95 \%$ of the rotation speed at $2.2 R_{d}$, with the low end of the distribution populated by galaxies with large bulges, which would be absent from our sample. 

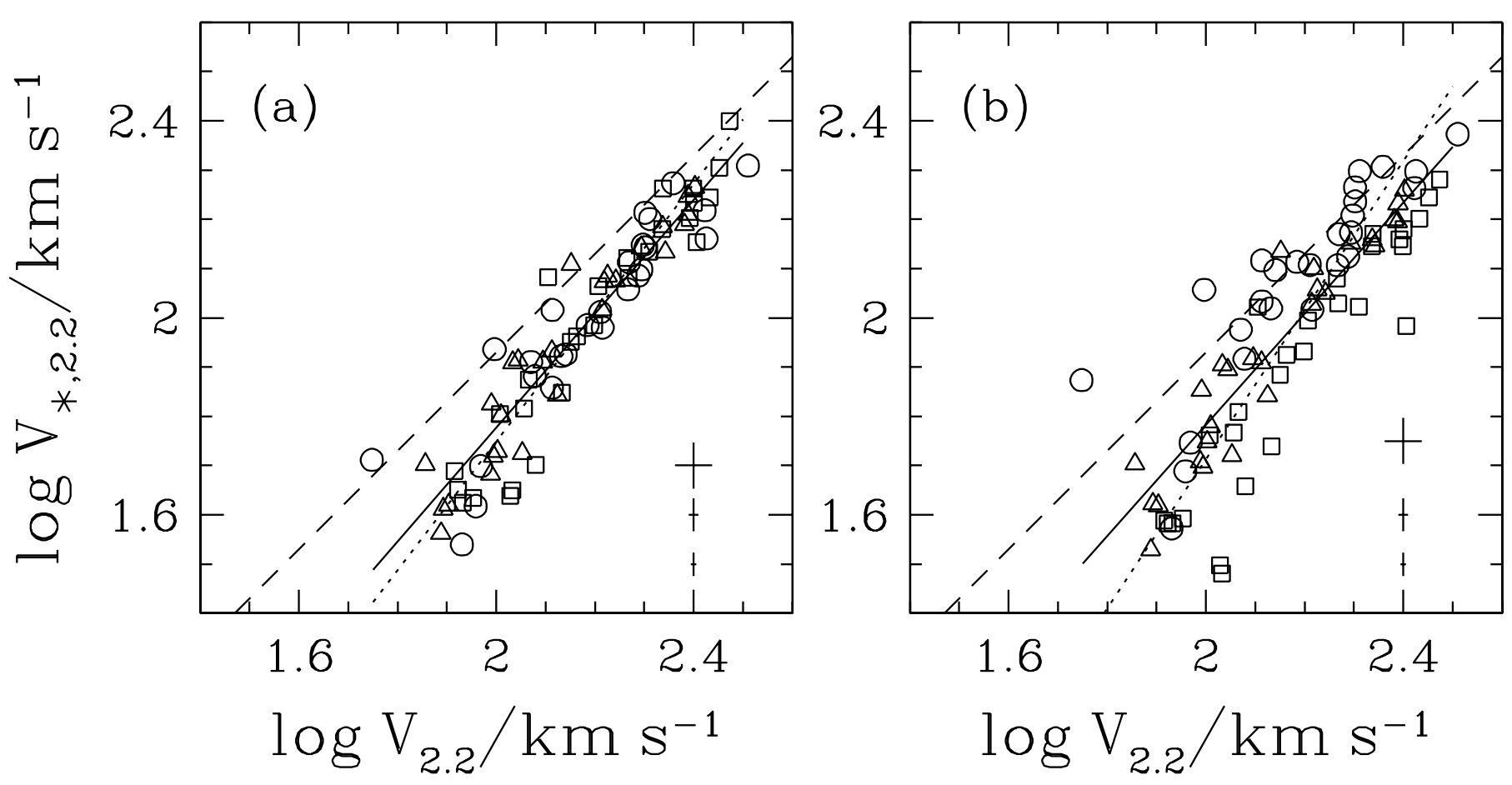

FIG. 5.-Comparison of the rotation velocity $V_{*, 2.2}$ predicted for each galaxy's stellar component (eq. [8]) to the observed $V_{2.2}$. Points are coded by residual from the $R_{d}-M_{*}$ relation, as in Fig. 3. In panel $(a), V_{* 2.2}$ is computed using the mean $R_{d}$ at each galaxy's $M_{*}$, while in panel $(b)$ it is computed using each galaxy's observed

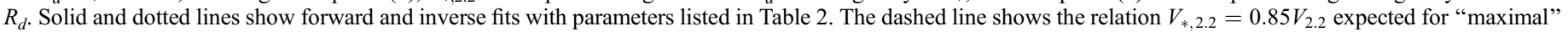
disks (Sackett 1997).

Our direct estimates with the Kroupa (2002) IMF lie below the maximal disk prediction, and they would continue to do so with the Kannappan (2004) gas correction. The increase of scatter between Figures $5 a$ and $5 b$, another manifestation of the uncorrelated TF77 and disk size residuals, implies that this gap is not simply a consequence of underestimating $M_{*} / L$ ratios; dark halos must provide an important contribution to rotational support at $2.2 R_{d}$.

Figure $6 a$ plots the inferred ratio of the total mass within 2.2 disk scale lengths to the stellar mass within 2.2 disk scale
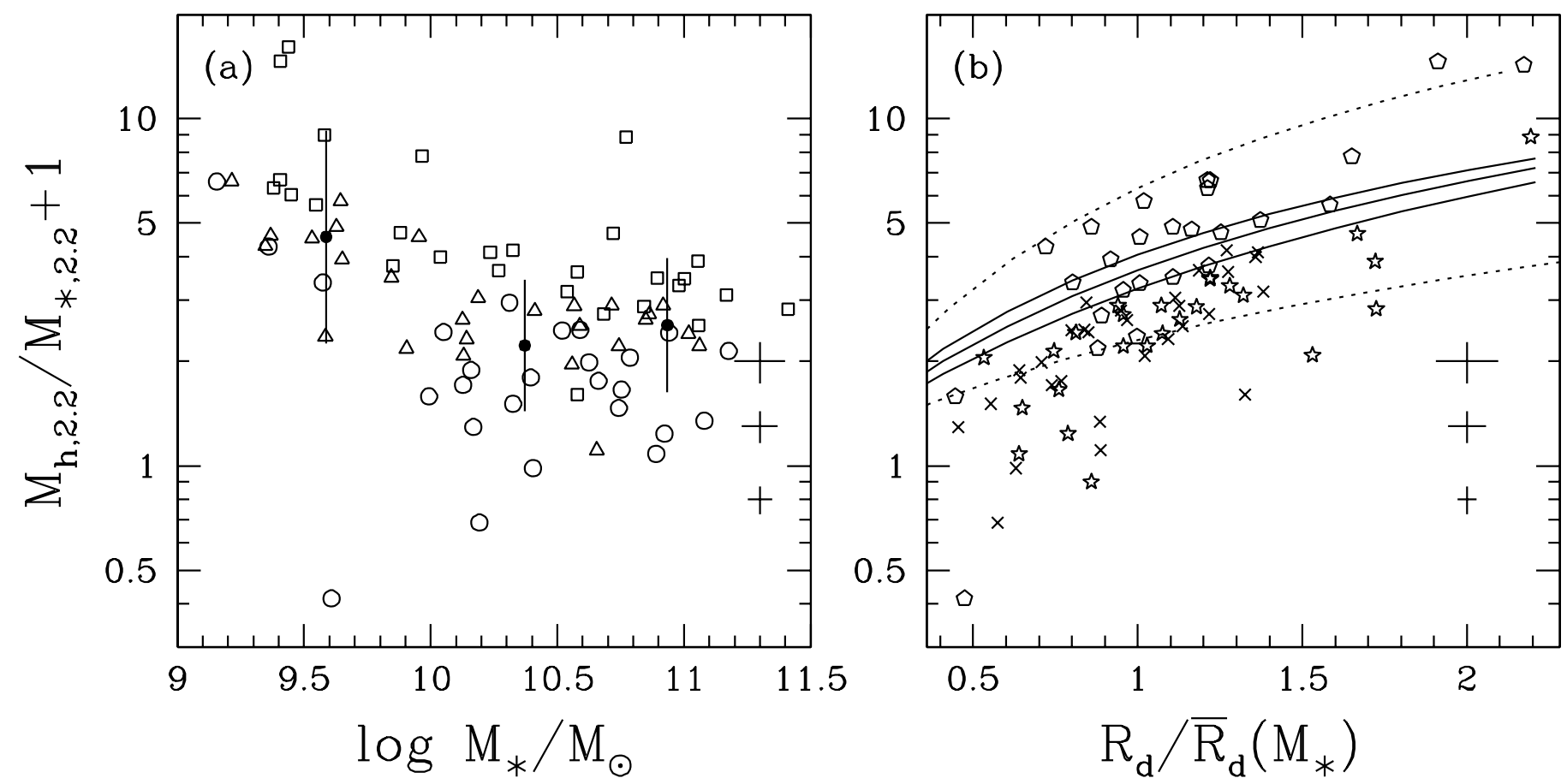

FIG. 6.- (a) Ratio of total mass within $2.2 R_{d}$ to stellar mass within $2.2 R_{d}$, as a function of $M_{*}$. Points are coded by residual from the $R_{d}-M_{*}$ relation. Filled circles with error bars show the mean and standard deviation for galaxies in the mass ranges $\log \left(M_{*} / M_{\odot}\right)>10.7,10-10.7$, and $<10$. $(b)$ Similar to $(a)$, but mass ratios are now plotted against the ratio of each galaxy's scale length to the mean scale length for its stellar mass. Crosses, stars, and pentagons show galaxies with

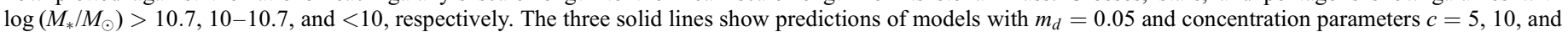
20 (bottom to top). The dotted lines show predictions for $m_{d}=0.025$ (upper) and 0.1 (lower), for $c=10$. 
lengths, as a function of stellar mass. The total mass is $M_{h, 2.2}+M_{*, 2.2}$ with

$$
M_{h, 2.2}=\frac{2.2 R_{d}}{G}\left(V_{2.2}^{2}-V_{*, 2.2}^{2}\right),
$$

$M_{*, 2,2}=0.65 f_{d} M_{*}+\left(1-f_{d}\right) M_{*}$, and $V_{*, 2,2}$ given by equation (8). The total-to-stellar mass ratio has a flat trend with considerable scatter for $M_{*} \geq 10^{10} M_{\odot}$, with a median value of $\sim 2.4$. For $M_{*}<10^{10} M_{\odot}$, the median ratio is higher $(\sim 4.4)$ in agreement with previous results (e.g., Persic et al. 1996), and the scatter is larger. This increased mass ratio corresponds to the steeper than unit slope of the $V_{*, 2.2}-V_{2.2}$ relation in Figure 5; the increase is reduced but not eliminated if we use the Kannappan (2004) gas correction to estimate baryonic masses instead of stellar masses. Points in Figure $6 a$ are again coded by residual from the $R_{d}-M_{*}$ relation, and the separation of circles (compact galaxies) and squares (diffuse galaxies) shows that the larger galaxies, at fixed $M_{*}$, have higher halo-to-stellar mass ratios within $2.2 R_{d}$, as one might expect.

While the scatter in disk sizes explains some of the scatter in $M_{h, 2.2} / M_{*, 2.2}$, Figure $6 b$ shows that much of the scatter must arise from another source. Here we plot $M_{h, 2.2} / M_{*, 2.2}$ against $R_{d} / \bar{R}_{d}\left(M_{*}\right)$, where $\bar{R}_{d}\left(M_{*}\right)$ is the mean disk scale length at the galaxy's stellar mass based on the best-fit $R_{d}-M_{*}$ relation (Fig. 3, dotted line). Points are now coded by galaxy stellar mass, with $M_{*}<10^{10} M_{\odot}$ shown by pentagons, $M_{*}>10^{10.7} M_{\odot}$ shown by stars, and intermediate-mass objects shown by crosses. At a fixed $R_{d} / \vec{R}_{d}\left(M_{*}\right)$, there is substantial scatter in $M_{h, 2.2} / M_{*, 2.2}$. The systematically higher halo-to-stellar mass ratios of low-mass galaxies are even clearer here than in Figure $6 a$.

The three solid lines in Figure $6 b$ show the predictions of models with $m_{d}=0.05$ and NFW halo concentrations of $c=5$, 10 , and 20 . Along each sequence, the value of $\lambda$ determines the value of $R_{d}$, and we set $\bar{R}_{d}\left(M_{*}\right)$ to be the model prediction for $\lambda=0.06$, tracking the central solid line of Figure 3. For these calculations we have assumed $M_{*}=10^{10} M_{\odot}$, but the lines are the same for any choice of $M_{*}$. To the extent that there is a central trend of the data points, the model lines describe it reasonably well, but it appears that a range of halo concentrations cannot account for the large scatter in $M_{h, 2.2} / M_{*, 2.2}$ at fixed disk size. The upper and lower dotted lines show predictions for $m_{d}=0.025$ and 0.10 , respectively, with $c=10$ and $\bar{R}_{d}\left(M_{*}\right)$ computed assuming $\lambda=0.045$ (for $m_{d}=0.025$ ) and $\lambda=0.08$ (for $m_{d}=0.10$ ). The envelope of these curves contains most of the data points, although there are a few with lower dark matter fractions. In the context of MMW98-style disk galaxy models, where $m_{d}, c$, and $\lambda$ are the parameters that determine disk properties, a substantial spread in $m_{d}$ is required to explain the observed distribution of galaxies in the $\left(M_{*}, V_{2.2}\right.$, $R_{d}$ )-space. In principle, the rotation curve shape can provide additional constraints on the disk mass fraction and its variation with galaxy properties (e.g., Persic \& Salucci 1988; Persic et al. 1996). However, our rotation curves are not very well resolved spatially, and modeling rotation curve shapes requires specific assumptions about dark halo profiles, so we have not attempted to exploit these constraints here.

Zavala et al. (2003) analyze the ratio of total mass to baryonic mass in a literature sample of disk galaxies and conclude that it correlates more directly with surface mass density than with luminosity or scale length individually. Figure 7 plots this correlation for our data set, using the mean stellar surface density within 2.2 scale lengths as the surface density measure. There is indeed a steady correlation over nearly 2 orders of

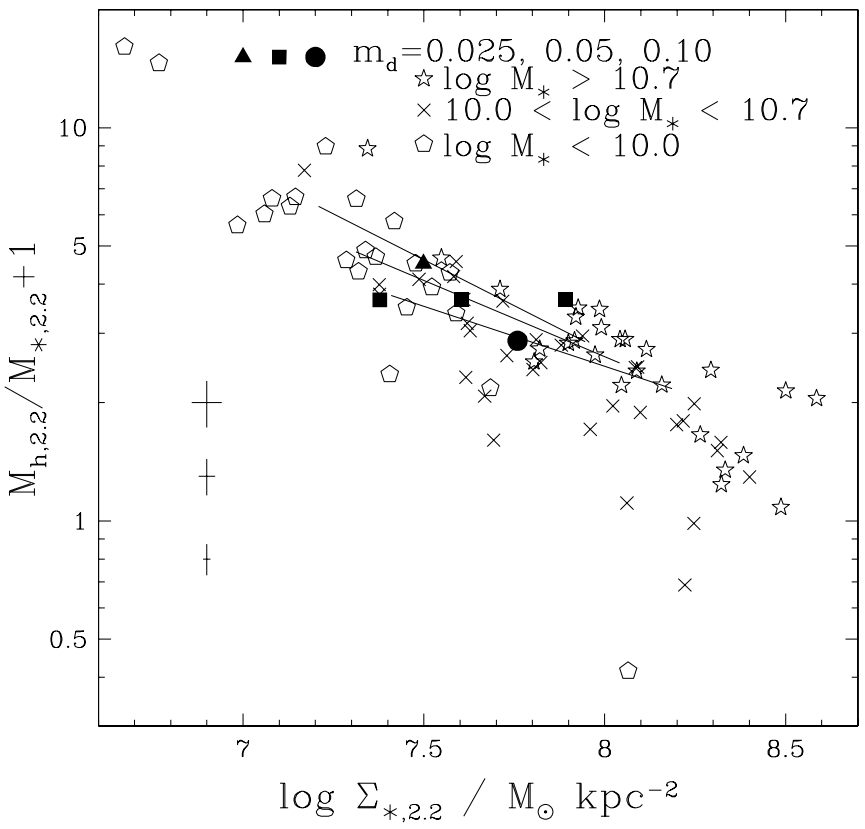

FIG. 7.- Total-to-stellar mass ratio as a function of the mean stellar surface density within $2.2 R_{d}$. Stars, crosses, and pentagons represent high-, intermediate-, and low-mass galaxies, respectively, as in Fig. $6 b$. The filled triangle, square, and circle with attached diagonal lines represent models with $\left(m_{d}, \lambda\right)=$ $(0.025,0.045),(0.05,0.06)$, and $(0.10,0.08)$, respectively, with $c=10$ and a halo mass $M_{h}=1.89 \times 10^{11} M_{\odot}$. The points correspond to the three central models shown in Fig. 4 , and lines show the effect of varying $\lambda$ over the range that reproduces the observed range of $R_{d}$, as in Fig. 4. Additional filled squares show the $m_{d}=0.05$ model for $M_{h}=4 \times 10^{10}\left(\operatorname{low} \Sigma_{*}\right)$ and $1.39 \times 10^{12} M_{\odot}\left(\right.$ high $\left.\Sigma_{*}\right)$; the lines of varying $\lambda$ would approximately parallel those of the central model.

magnitude in surface density, somewhat tighter than the correlations in Figure 6, although still with significant scatter and occasional large outliers. Most significantly, one can see that the total-to-stellar mass ratios for low surface density, highmass galaxies are similar to those of typical low-mass galaxies with similar surface density. This result suggests, as argued by Zavala et al. (2003), that the dependencies of the mass ratio on galaxy mass and scale length can be understood as largely reflecting a more fundamental dependence on surface density. The distribution of our data points in Figure 7 is similar to the distribution found by Zavala et al. (2003), although their sample is constructed to include more low surface brightness galaxies.

Large filled squares show model predictions for $m_{d}=0.05$, halo masses $M_{h}=4 \times 10^{10}, 1.89 \times 10^{11}$, and $1.39 \times 10^{12} M_{\odot}$, and spin parameter $\lambda=0.06$. Diagonal lines attached to these points span the range $\lambda=0.04-0.08$, with low-spin disks having high $\Sigma_{*}$ and low $M_{h, 2.2} / M_{*, 2.2}$. Filled triangles and circles show corresponding predictions for $m_{d}=0.025$ and 0.05 , with halo mass $1.89 \times 10^{11} M_{\odot}$ and the $\lambda$-values that reproduce the three $R_{d}-M_{*}$ lines in Figure 3. The data roughly follow the trend predicted for $\lambda$ variations or $m_{d}$ variations as drivers of surface brightness variations. At fixed $m_{d}$ and $\lambda$, a change of halo mass does not change $M_{h, 2.2} / M_{*, 2.2}$, so the continuity of the trend for different mass galaxies again suggests that high-mass galaxies have higher $m_{d}$, not simply higher $M_{h}$.

\section{SUMMARY AND DISCUSSION}

We have examined the correlations among stellar mass, disk scale length, and rotation velocity at $2.2 R_{d}$ for a sample of 81 disk-dominated galaxies selected from the SDSS main galaxy redshift sample. The SDSS selection allows us to choose systems 
with a roughly flat distribution of absolute magnitude over the range $-18 \leq M_{r} \leq-22$ at redshifts such that peculiar velocities induce relatively small distance uncertainties. SDSS multicolor photometry allows us to assign stellar masses to galaxies based on their $i$-band luminosities and $g-r$ colors, using the prescription of Bell et al. (2003) converted to a Kroupa (2002) IMF. We use the SDSS $i$-band images to perform two-dimensional bulge-disk decomposition with GALFIT (Peng et al. 2002). The defining morphological characteristics of our sample are SDSS isophotal axis ratio $b / a<0.6$ and GALFIT $i$-band disk-to-total luminosity ratios $f_{d} \geq 0.9$. We do not apply any environmental preselection, so our sample should be representative of the range of environments in which galaxies of these morphological characteristics and absolute magnitudes are found.

Our principal observational results are as follows:

1. We find a best-fit (forward) $i$-band TF77 relation $\left(L_{i} / 10^{10} L_{\odot}\right)=1.84\left(V_{2.2} / 150 \mathrm{~km} \mathrm{~s}^{-1}\right)^{2.60}$, with an estimated intrinsic scatter of $0.13 \mathrm{dex}$, or $0.33 \mathrm{mag}$. The slope is shallower than that found by some previous analyses (e.g., Verheijen 2001; Kannappan et al. 2002) but is similar to that of Courteau (1997), whose sample selection and analysis methods are closest to those here. Possible sources of difference include the environmental properties of the sample ("field" vs. "cluster"), the morphological criteria (disk/total $\geq 0.9$ vs. more general "disk galaxy" selection), the adopted velocity measure ( $V_{2.2}$ vs. $V_{\text {flat }}$ or $\mathrm{H}_{\mathrm{I}}$ line width), and the fitting procedures. The intrinsic scatter is similar to that found for previous samples, although it rises substantially if we do not restrict the sample to diskdominated galaxies (see P05). There is a weak trend for galaxies with redder than average colors to lie below the best-fit TF77 relation (low $L_{i}$ ).

2. We find a best-fit (forward) stellar mass TF77 relation $\left(M_{*} / 10^{10} M_{*}\right)=2.32\left(V_{2.2} / 156 \mathrm{~km} \mathrm{~s}^{-1}\right)^{3.05}$, with an estimated intrinsic scatter of $0.16 \mathrm{dex}$. The use of stellar mass removes the weak trend with color residual, suggesting that stellar mass plays a more fundamental role than luminosity in TF77 correlations. The slope is shallower than that found by BD01 for Ursa Major spiral galaxies, with good agreement at $V_{2.2} \sim 200 \mathrm{~km} \mathrm{~s}^{-1}$ but higher $M_{*}$ in our sample at $V_{2.2} \sim 100 \mathrm{~km} \mathrm{~s}^{-1}$. Possible sources of difference again include a range of environments, morphological criteria, velocity measure, and fitting procedures. Our relation is also shallower than the baryonic mass TF77 relation of McGaugh et al. (2000), whose sample is more heterogeneous and extends to lower luminosities. For our sample, a statistical, color-based correction for gas-to-stellar mass fractions (Kannappan 2004) makes only a modest difference to the TF77 parameters.

3. The $R_{d}-M_{*}$ distribution has a best-fit mean relation $R_{d}=$ $4.0\left(M_{*} / 2.2 \times 10^{10} M_{\odot}\right)^{0.24} \mathrm{kpc}$, but the distribution is broad, with roughly a factor of 3 range in disk scale length at fixed $M_{*}$.

4. There is no discernible correlation between residuals of the $R_{d}-M_{*}$ relation and residuals of the $M_{*}-V_{2.2}$ relation. At a given $M_{*}$, compact galaxies do not rotate faster or slower than average. This result agrees with earlier analyses showing weak or negligible trends of TF77 residual with disk scale length or surface brightness (Zwaan et al. 1995; Courteau \& Rix 1999; Verheijen 2001). As emphasized by Courteau \& Rix (1999), the lack of correlation between TF77 residual and disk size implies that disks cannot, in most cases, make a dominant contribution to rotation velocities at $2.2 R_{d}$. This evidence for "submaximal" disks agrees with independent arguments based on disk scale heights and vertical velocity dispersions (Bottema 1993, 1995; Kregel et al. 2005).
5. Direct estimates of the stellar contribution to the rotation speed at $2.2 R_{d}$, based on the population synthesis mass-tolight ratios for a Kroupa (2002) IMF, yield a best-fit relation $\left(V_{*, 2.2} / 156 \mathrm{~km} \mathrm{~s}^{-1}\right)=0.64\left(V_{2.2} / 156 \mathrm{~km} \mathrm{~s}^{-1}\right)^{1.16}$. Including estimated gas masses following Kannappan (2004) changes the intercept to 0.71 and the slope to 1.07 . The low contribution from the stellar disk is consistent with the weak $R_{d}$ trend noted above, and it agrees well with the Kregel et al. (2005) estimate of $0.53 \pm 0.04$ based on disk scale heights. Courteau \& Rix (1999) give an estimate of 0.6 , also in good agreement with our results. The scatter between $V_{*, 2.2}$ and $V_{2.2}$ is larger ( 0.11 vs. $\left.0.06 \mathrm{dex}\right)$ if we use each galaxy's actual $R_{d}$ instead of the mean $R_{d}$ at the galaxy's $M_{*}$, another sign of the weak correlation between size and rotation speed at fixed $M_{*}$.

6. The ratio of halo to stellar mass within $2.2 R_{d}$ has a large range at a given $M_{*}$. Median ratios are 2.4 for galaxies with $M_{*}>10^{10} M_{\odot}$ and 4.4 for $10^{9} M_{\odot}<M_{*}<10^{10} M_{\odot}$. At any $M_{*}$, compact galaxies have lower $M_{h, 2.2} / M_{*, 2.2}$ and large galaxies have higher $M_{h, 2.2} / M_{*, 2.2}$, as expected. However, the spread in radius accounts for only a fraction of the scatter in halo-to-stellar mass ratio. Galaxies with a wide range of $M_{*}$ and $R_{d}$ trace out a continuous correlation between halo-to-stellar mass ratio and disk surface density, in agreement with Zavala et al. (2003), but the scatter about the mean trend is substantial.

For early-type galaxies, the "fundamental plane" is close to a virial relation for the stellar component, $\sigma^{2} \sim G M_{*} / R_{\text {eff }}$ (Djorgovski \& Davis 1987; Dressler et al. 1987; Bernardi et al. 2002), and the scatter of the bivariate $L-\sigma$ relation is significantly larger than the scatter about the fundamental plane. The nearvirial form is naturally explained if stars dominate the central gravitational potential that determines the observed velocity dispersion, with only a modest contribution from dark matter (see Rusin \& Kochanek 2005 and references therein). For disk galaxies, our results show the opposite: $V_{2.2}$ is better correlated with $M_{*}$ (or $L$ ) than with $G M_{*} / R_{d}$, making the $M_{*}-V_{2.2}$ an essentially edge-on view of the disk galaxy fundamental plane. The minimal effect of disk size on $V_{2.2}$ implies that dark matter must contribute a large fraction of the mass within the central 2 scale lengths of disk galaxies, in accord with our direct (but IMF dependent) inference based on stellar mass-to-light ratios. Our conclusions on these points agree with those of Courteau \& Rix (1999) and with the more recent analysis of Courteau et al. (2003), who investigate the scaling relations of disk galaxy properties in a larger but less tightly defined sample.

To put our observational results in context, we have compared them to the predictions of theoretical models in which disks form by the dissipative collapse of gas in cold dark matter halos (Fall \& Efstathiou 1980; Dalcanton et al. 1997; MMW98; Shen et al. 2002; Dutton et al. 2005). The scale length and rotation speed of a galaxy with specified $M_{*}$ are determined in these models by the ratio of disk mass to halo virial mass $\left(m_{d}\right)$, the spin parameter $(\lambda)$, and the concentration parameter $(c)$ of the NFW halo. In practice, variations of $c$ within the expected range have only moderate impact on $R_{d}$ and $V_{2.2}$, in part because adiabatic contraction alters the inner regions of the dark matter halo. A model with $m_{d}=0.05$ reproduces the observed $R_{d}-M_{*}$ distribution if $\lambda$-values range from $\sim 0.04$ to $\sim 0.08$, a span that omits the lower half and the extreme upper tail of the lognormal $\lambda$ distribution predicted for dark matter halos (e.g., Bullock et al. 2001). For $m_{d}=0.025$ and 0.10 , the required values of $\lambda$ are, respectively, lower by $25 \%$ and higher by $\sim 30 \%$.

The $m_{d}=0.05$ model reproduces our measured $M_{*}-V_{2.2}$ relation reasonably well, and it predicts a weak correlation between 
size and TF77 residual that could plausibly be washed out by the TF77 scatter. (Note, however, that such a model with fixed $m_{d}$ does not explain the magnitude of the inferred intrinsic scatter.) The $m_{d}=0.025$ model predicts lower $M_{*}$ at a given $V_{2.2}$, so it would require a different IMF (with fewer low-mass stars) to be consistent with our data. The $m_{d}=0.10$ model predicts slightly higher $M_{*}$ values at a given $V_{2.2}$, and it predicts substantial size-TF77 residual correlations because of its high-mass disks. These strong residual correlations appear incompatible with our data.

The model comparisons in Figure $6 b$ suggest that no model with a single value of $m_{d}$ will reproduce our observed distribution of data points: the large scatter in $M_{h, 2.2} / M_{*, 2.2}$ at fixed $R_{d} / \bar{R}_{d}\left(M_{*}\right)$ can only be explained with scatter in the ratio of stellar mass to total halo mass. For our Kroupa (2002) IMF normalization, Figure $6 b$ suggests $m_{d}$ values in the range $\sim 0.025-0.05$ for galaxies with $10^{9} M_{\odot}<M_{*}<10^{10} M_{\odot}$ and $\sim 0.05-0.10$ for galaxies with $M_{*} \geq 10^{10} M_{\odot}$. Systematically lower $m_{d}$ values for low-mass galaxies are a plausible signature of more efficient supernova feedback in shallow gravitational potential wells (Dekel \& Silk 1986) or in galaxies that lack a confining envelope of shock-heated gas (Keres et al. 2004).

We have not attempted to develop a complete and selfconsistent model for the distribution of disk mass functions and halo parameters needed to reproduce the observed, joint distribution of $M_{*}, R_{d}$, and $V_{2.2}$. The results above suggest the outline of such a model: disk mass fractions span a substantial range, with a central value $m_{d} \sim 0.05$ and systematically lower values for low-mass galaxies, and the disk galaxy population samples mainly the upper half of the halo spin parameter distribution. Our observations provide the data needed to constrain more complete models of this sort, along the lines pursued by Shen et al. (2002) and Dutton et al. (2005), or to test the predictions of hydrodynamic simulations of disk galaxy formation (e.g., Navarro \& Steinmetz 1997). Confrontations between these models and our data should lead to better understanding of the mechanisms that govern disk galaxy formation and the relations between dark and luminous matter in the inner regions of disk galaxies.

We thank Richard Pogge for assistance with the MDM observations and Oleg Gnedin for helpful discussions and for providing his adiabatic contraction code. We also thank Michael Blanton and David Hogg for comments on the manuscript. J. P. and D. W. acknowledge support from NSF grants AST 00-98584 and AST 04-07125.

Funding for the creation and distribution of the SDSS Archive has been provided by the Alfred P. Sloan Foundation, the Participating Institutions, the National Aeronautics and Space Administration, the National Science Foundation, the US Department of Energy, the Japanese Monbukagakusho, and the Max Planck Society. The SDSS Web site is http://www.sdss.org.

The SDSS is managed by the Astrophysical Research Consortium (ARC) for the Participating Institutions. The Participating Institutions are the University of Chicago, Fermilab, the Institute for Advanced Study, the Japan Participation Group, Johns Hopkins University, the Korean Scientist Group, Los Alamos National Laboratory, the Max-Planck-Institute for Astronomy (MPIA), the Max-Planck-Institute for Astrophysics (MPA), New Mexico State University, University of Pittsburgh, University of Portsmouth, Princeton University, the US Naval Observatory, and the University of Washington.

This paper is based in part on observations obtained in the framework of the Calar Alto Key Project for SDSS Follow-up Observations (Grebel 2001) at the German-Spanish Astronomical Centre, Calar Alto Observatory, operated by the MaxPlanck-Institute for Astronomy, Heidelberg, jointly with the Spanish National Commission for Astronomy.

\section{APPENDIX}

Random errors in galaxy distances or magnitudes can lead to biased estimates of TF77 parameters because there are more distant and faint galaxies to scatter in one direction than vice versa and because objects can scatter across selection boundaries. Most discussions of these "Malmquist"-type biases have focused on apparent magnitude-limited samples, or on the systematic bias in the derived peculiar velocity field (e.g., Lynden-Bell et al. 1988; Gould 1993; Teerikorpi 1993; Strauss \& Willick 1995). Since our selection procedure is quite different from those of most previous TF77 surveys, we have tested for Malmquist-type biases with a simple Monte Carlo experiment.

The measurement errors in SDSS Petrosian magnitudes are generally small, and luminosity uncertainties are therefore dominated by line-of-sight peculiar velocities, which we have assumed in our analysis to be drawn from a Gaussian of dispersion $300 \mathrm{~km} \mathrm{~s}^{-1}$. For our Monte Carlo sample, we assign random three-dimensional positions to $10^{6}$ artificial galaxies and draw their absolute $r$-band magnitudes from the SDSS luminosity function of Blanton et al. (2003c). We assign each of these galaxies a circular velocity drawn from the best-fit $i$-band inverse TF77 relation listed in Table 2, with slope $a=0.346$, intercept $b=\log \left(V_{2.2} / \mathrm{km} \mathrm{s}^{-1}\right)=2.185$ at $\log \left(L / L_{\odot}\right)=10.293$, and intrinsic scatter of $\sigma=0.048 \mathrm{dex}$ in $\log V_{2.2}$. For purposes of this experiment, we ignore the slight difference between $i$-band and $r$-band TF77 relations, since we only need a qualitatively realistic assignment for the test.

We modify each galaxy's redshift by a peculiar velocity drawn from a $300 \mathrm{~km} \mathrm{~s}^{-1}$ Gaussian, and we apply the same absolute magnitude-dependent redshift cuts that we used for our sample definition (see $\S 2$ ), using the apparent rather than true absolute magnitude. Finally, we randomly draw from this cut sample a subset of galaxies that matches the nearly flat $M_{r}$ distribution of our observed sample, selecting 10 times as many artificial galaxies as observed galaxies in each absolute magnitude bin. Applying our maximum likelihood estimation method to this artificial sample yields inverse TF77 parameters $a=0.347, b=2.184$, and $\sigma=0.050$, in excellent agreement with our input values. The forward TF77 parameters are $a=2.613, b=10.26$, and $\sigma=0.14$ mag, in excellent agreement with the values of $2.603 \pm 0.133,10.266 \pm 0.020$, and $0.131 \pm 0.015$ derived for our observed sample (see Table 2). We conclude that any Malmquist-type biases in our sample definition or analysis are smaller than our quoted statistical errors. This small impact is not surprising, since the fractional distance errors are small and the velocity and absolute magnitude range of the sample are fairly large, making scatter across selection boundaries a minimal effect. 
Abazajian, K., et al. 2004, AJ, 128, 502

Avila-Reese, V., Firmani, C., \& Hernandez, X. 1998, ApJ, 505, 37

Bell, E. F., \& de Jong, R. S. 2001, ApJ, 550, 212 (BD01)

Bell, E. F., McIntosh, D. H., Katz, N., \& Weinberg, M. D. 2003, ApJS, 149, 289

Bernardi, M., Alonso, M. V., da Costa, L. N., Willmer, C. N. A., Wegner, G., Pellegrini, P. S., Rité, C., \& Maia, M. A. G. 2002, AJ, 123, 2159

Binney, J., \& Tremaine, S. 1987, Galactic Dynamics (Princeton: Princeton Univ. Press)

Blanton, M. R., Lupton, R. H., Maley, F. M., Young, N., Zehavi, I., \& Loveday, J. 2003a, AJ, 125, 2276

Blanton, M. R., et al. 2003b, AJ, 125, 2348 2003c, ApJ, 592, 819

Bottema, R. 1993, A\&A, 275, 16 1995, A\&A, 295, 605

Bullock, J. S., Kolatt, T. S., Kravstov, A. V., Klypin, A. A., Porciani, C., \& Primack, J. R. 2001, ApJ, 555, 240

Cole, S., Aragon-Salamanca, A., Frenk, C. S., Navarro, J. F., \& Zepf, S. E. 1994, MNRAS, 271, 781

Cole, S., \& Kaiser, N. 1989, MNRAS, 237, 1127

Courteau, S. 1997, AJ, 114, 2402

Courteau, S., MacArthur, L. A., Dekel, A., van den Bosch, F., McIntosh, D. H., \& Dale, D. 2003, ApJ, submitted (astro-ph/0310440)

Courteau, S., \& Rix, H.-W. 1999, ApJ, 513, 561

Dalcanton, J. J., Spergel, D. N., \& Summers, F. J. 1997, ApJ, 482, 659

Dekel, A., \& Silk, J. 1986, ApJ, 303, 39

Djorgovski, S., \& Davis, M. 1987, ApJ, 313, 59

Dressler, A., Lynden-Bell, D., Burstein, D., Davies, R. L., Faber, S. M., Terlevich, R., \& Wegner, G. 1987, ApJ, 313, 42

Dutton, A. A., van den Bosch, F. C., Courteau, S., \& Dekel, A. 2005, in Baryons in Dark Matter Halos, ed. R.-J. Dettmar, U. Klein, \& P. Salucci, in press (astro-ph/0501256)

Eisenstein, D. J., \& Loeb, A. 1996, ApJ, 459, 432

Fall, S. M., \& Efstathiou, G. 1980, MNRAS, 193, 189

Firmani, C., \& Avila-Reese, V. 2000, MNRAS, 315, 457

Freeman, K. C. 1970 , ApJ, 160, 811

Fukugita, M., Ichikawa, T., Gunn, J. E., Doi, M., Shimasaku, K., \& Schneider, D. P. 1996, AJ, 111, 1748

Gnedin, O. Y., Kravstov, A. V., Klypin, A. A., \& Nagai, D. 2004, ApJ, 616, 16

Gould, A. 1993, ApJ, 412, L55

Graham, A. W., Driver, S. P., Petrosian, V., Conselice, C. J., Bershady, M. A., Crawford, S. M., \& Goto, T. 2005, AJ, 130, 1535

Grebel, E. K. 2001, Rev. Mod. Astron., 14, 223

Gunn, J. E. 1983, in IAU Symp. 100, Internal Kinematics and Dynamics of Galaxies, ed. E. Athanassoula (Dordrecht: Reidel), 379

Gunn, J. E., et al. 1998, AJ, 116, 3040

Haynes, M. P., \& Giovanelli, R. 1984, AJ, 89, 758

Hogg, D. W., Schlegel, D. J., Finkbeiner, D. P., \& Gunn, J. E. 2001, AJ, 122, 2129

Ivezić, Ž., et al. 2004, Astron. Nachr., 325, 583

\section{EFERENCES}

Kannappan, S. J. 2004, ApJ, 611, L89

Kannappan, S. J., Fabricant, D. G., \& Franx, M. 2002, AJ, 123, 2358

Kauffmann, G., White, S. D. M., \& Guiderdoni, B. 1993, MNRAS, 264, 201

Kauffmann, G., et al. 2003, MNRAS, 341, 33

Keres, D., Katz, N., Davé, R., \& Weinberg, D. H. 2004, MNRAS, 363, 2

Kregel, M., van der Kruit, P. C., \& Freeman, K. C. 2005, MNRAS, 358, 503 Kroupa, P. 2002, Science, 295, 82

Lupton, R., Gunn, J. E., Ivezić, Ž., Knapp, G. R., Kent, S., \& Yasuda, N. 2001, in ASP Conf. Ser. 238, Astronomical Data Analysis Software and Systems X, ed. F. R. Harnden, Jr., F. A. Primini, \& H. E. Payne (San Francisco: ASP), 269

Lynden-Bell, D., Faber, S. M., Burstein, D., Davies, R. L., Dressler, A., Terlevich, R. J., \& Wegner, G. 1988, ApJ, 326, 19

Massey, P., Valdes, F., \& Barnes, J. 1992, A User's Guide to Reducing Slit Spectra with IRAF (Tucson: NOAO), http://iraf.noao.edu/iraf/ftp/iraf/docs/ spect.ps.Z

McGaugh, S. S., Schombert, J. M., Bothun, G. D., \& de Blok, W. J. G. 2000, ApJ, 533, L99

Mo, H. J., \& Mao, S. 2000, MNRAS, 318, 163

Mo, H. J., Mao, S., \& White, S. D. M. 1998, MNRAS, 295, 319 (MMW98)

Navarro, J. F., Frenk, C. S., \& White, S. D. M. 1997, ApJ, 490, 493

Navarro, J. F., \& Steinmetz, M. 1997, ApJ, 478, 13

Peng, C. Y., Ho, L. C., Impey, C. D., \& Rix, H.-W. 2002, AJ, 124, 266

Persic, M., \& Salucci, P. 1988, MNRAS, 234, 131

Persic, M., Salucci, P., \& Stel, F. 1996, MNRAS, 281, 27

Pier, J. R., Munn, J. A., Hindsley, R. B., Hennessy, G. S., Kent, S. M., Lupton, R. H., \& Ivezić, Ž. 2003, AJ, 125, 1559

Press, W. H., Teukolsky, S. A., Vetterling, W. T., \& Flannery, B. P. 1992, Numerical Recipes in FORTRAN (Cambridge: Cambridge Univ. Press)

Rusin, D., \& Kochanek, C. S. 2005, ApJ, 623, 666

Sackett, P. D. 1997, ApJ, 483, 103

Sersić, J. L. 1968, Atlas de Galaxias Australes (Cordoba: Obs. Astron.)

Shen, S., Mo, H. J., \& Shu, C. 2002, MNRAS, 331, 259

Smith, J. A., et al. 2002, AJ, 123, 2121

Steinmetz, M., \& Navarro, J. F. 1999, ApJ, 513, 555

Stoughton, C., et al. 2002, AJ, 123, 485

Strauss, M. A., \& Willick, J. A. 1995, Phys. Rep., 261, 271

Strauss, M. A., et al. 2002, AJ, 124, 1810

Teerikorpi, P. 1993, A\&A, 280, 443

Tully, R. B., \& Fisher, J. R. 1977, A\&A, 54, 661 (TF77)

Tully, R. B., Pierce, M. J., Huang, J.-S., Saunders, W., Verheijen, M. A. W., \& Witchalls, P. L. 1998, AJ, 115, 2264

Verheijen, M. A. W. 2001, ApJ, 563, 694

Willick, J. A., Strauss, M. A., Dekel, A., \& Kolatt, T. 1997, ApJ, 486, 629

York, D. G., et al. 2000, AJ, 120, 1579

Zavala, J., Avila-Reese, V., Hernández-Toledo, H., \& Firmani, C. 2003, A\&A, 412,633

Zwaan, M. A., van der Hulst, J. M., de Blok, W. J. G., \& McGaugh, S. S. 1995, MNRAS, 273, L35 